Article

\title{
Numerical Simulations of a Gas-Solid Two-Phase Impinging Stream Reactor with Dynamic Inlet Flow
}

\author{
Xueqing Liu ${ }^{1}$, Song Yue ${ }^{2}$, Luyi Lu ${ }^{1, *}$, Wei Gao ${ }^{1}$ and Jianlan $\mathrm{Li}^{1}$ \\ 1 School of Energy and Power Engineering, Huazhong University of Science and Technology, \\ 1037 Luoyu Road, Wuhan 430074, China; d201677300@hust.edu.cn (X.L.); gw@hust.edu.cn (W.G.); \\ hust_lil@hust.edu.cn (J.L.) \\ 2 Power Generation Branch, POWERCHINA Hubei Electric Engineering Corporation Limited, \\ No.1 Xinqiaosi Road, Jinyinhu Street, Dongxihu District, Wuhan 430040, China; \\ yuesong-huby@powerchina.cn \\ * Correspondence: hust_lly@hust.edu.cn; Tel.: +86-135-5408-9897
}

Received: 5 June 2018; Accepted: 19 July 2018; Published: 23 July 2018

\begin{abstract}
Fluid flow characteristics and particle motion behavior of an impinging stream reactor with dynamic inlet flow (both inlet velocity patterns exhibit step variation) are investigated and discussed with the computational fluid dynamics-discrete element method (CFD-DEM). The effect of $T$ (variation period of the dynamic inlet flow) and $\Delta u$ (inlet velocity difference) on the motion characteristics of single and multiple particles, as well as the mean particle residence time, are studied and discussed. The research results indicate that, compared with the traditional impinging stream reactor (both inlet velocities are equal and constant) with equal mean inlet velocity $\left(u_{m}\right)$ within one period, the impinging surface is instantaneously moving and the flow regime is varied with time in the impinging stream reactor with dynamic inlet flow. The impinging stream reactor with dynamic inlet flow provides higher cost performance over the traditional impinging stream reactor, under equal $u_{m}$, in terms of single-particle residence time. Moreover, three new particle motion modes exist in multi-particle motions of the impinging stream reactor with dynamic inlet flow; particles are accelerated by the original or reverse fluid and perform oscillatory motion at least once after an interparticle collision. Whether it is a single particle or multi-particles, the mean particle residence time reaches a maximum value when $T / 2$ is approximately equal to the first particle acceleration time, since the maximum axial kinetic energy increases in every oscillatory motion compared with traditional impinging stream, and the number of oscillatory motions is increasing. The mean residence time of a particle in the impinging stream reactor with a dynamic inlet flow increases with increasing $\Delta u$, since the dynamic inlet conditions and increasing $\Delta u$ can continuously supply more energy to particles and thus cause more particles to enter one of the three new modes of particle motion.
\end{abstract}

Keywords: impinging stream reactor; particle mean residence time; CFD-DEM; particle motion

\section{Introduction}

The impinging stream technique was first proposed by the Soviet scientist Elperin in 1961, and then investigated and discussed by many scholars and scientists [1-4]. The impinging stream reactor can effectively strengthen interphase heat and mass transfer, and thus is widely used in drying [5,6], absorption [7,8], micromixing [9-11], combustion [12], crystallization [13], extraction $[14,15]$, nanoparticles preparation $[16,17]$, and so on, which has created great potential for engineering applications.

The traditional coaxial impinging stream reactor, where both inlet fluid velocities are equal and constant, is widely used in practical applications. Many articles have numerically and experimentally 
investigated and discussed the fluid flow characteristics and regimes of the traditional impinging stream reactor. Huai et al. [18] investigated and discussed drying performance and fluid motion behavior in a semi-circular impinging stream reactor. Their results show that geometrical models and inlet flow conditions have a great impact on the drying efficiency and motion characteristics of wet materials. Wang et al. [19] performed many simulation studies to investigate and measure the mixing performance and fluid motion behavior of multiple and multi-set three-dimensional confined turbulent round opposing jets. They found that flow and mixing characteristics have a close relationship with the impinging stream configuration. Liu et al. [20] carried out many simulations to investigate and measure the gas flow characteristics and particle motion behavior in the impinging stream reactor. Their results show that two pairs of counter-rotating gas vortices and interparticle collisions in the impingement region shorten the particle residence time due to the entrainment of gas on the particle. Chicharron and Devahastin [21] performed many simulations on the motion behaviors of both fluids and particles in an impinging stream dryer by means of the computational fluid dynamics-discrete element method (CFD-DEM). In their work, the effect of the impinging spacing, inlet air velocity, and particle inlet flow rate on the flow characteristics and drying performance of the impinging stream reactor were studied and discussed. Li and Yao [22] experimentally studied the flow regime of the axisymmetric and planar impinging stream reactor by means of a smoke-wire technique. They performed a detailed analysis of the influence of nozzle structure, geometric configuration, and Reynolds numbers on the flow regime and characteristics of the impinging stream reactor. Zhang [23] and Liu performed many experimental studies on the flow characteristics in free impinging jet reactors by particle image velocimetry. They analyzed the effect of inlet parameters and the ratio of the jet distance to the jet diameter on the liquid flow behavior, turbulent kinetic energy distribution, and mixing efficiency of the impinging jet reactor. From the above literature review, it is evident that the flow characteristics of the impinging stream reactor are determined by the inflow conditions and the geometric model of the reactor. Thus, a better understanding of the particle residence time is necessary for optimization of these processes.

Meanwhile, some investigations have been conducted on particle motion behavior in the traditional impinging stream reactor. Although different simulation tools were used to investigate and measure detailed information of the flow field, without consideration of the interparticle interactions, the simulated data are in discrepancy with the experimental results. Jafarikojour et al. [24] studied the droplet residence time distribution in the impinging stream reactor by means of descending-sized stirred tanks in series, without consideration of the droplet interaction forces. Rajaie et al. [25] investigated and measured the residence time distribution of the aqueous phase and the velocity distribution of the flow field by means of a direct simulation Monte Carlo method, but again the interparticle interactions were neglected. Fatourehchi et al. [26] studied the residence time distribution in the impinging stream reactor by means of the gamma distribution model with bypass (GDB) without consideration of the interparticle forces. Wu et al. [27] numerically studied and discussed the particle motion behavior in an axisymmetric impinging stream reactor. In their work, interparticle collisions have a significant impact on the multi-particle motion of the flow field, including the particle motion trajectory and particle residence time. Khomwachirakul and Devahastin [6] investigated the gas and particle motion behavior and particle mean residence time in a coaxial impinging stream reactor, considering particle-particle interactions by CFD-DEM. In their work, the CFD-DEM model yielded some critical parameters mentioned above and made predictions of multiphase transport phenomena that agree well with experimental results. Du and Zhao [28] numerically studied and measured particle motion characteristic of the flow field with consideration of interparticle interactions. Their results indicated that most particles scatter in all directions after interparticle collisions in the impingement region, and few particles went into the opposite streams, performing deceleration motions, which greatly shorten the particle residence time and particle motion range. The short particle residence time of traditional impinging stream techniques can cause some relevant reactions to be inadequately carried out and greatly reduce the reaction effectiveness. 
In recent years, a few researchers have been paying attention to impinging stream reactors with dynamic inlet flow. Erkoc et al. [29] carried out many simulations on the mixing performance of reactants and fluid motion behavior in a confined T-jets reactor with the induction of resonance. Their results indicate that the flow regime, vortex motions, and flow characteristics in the impinging stream reactor have a close relationship with the inlet flow conditions, such as the frequency and amplitude of the inlet pulsation. Li et al. [30] experimentally investigated and measured the motion characteristics of the impinging surface and flow regime of the flow field in the axisymmetric turbulent impinging streams with a modulated airflow via a hot-wire anemometer and a flow visualization technique. In their work, the impinging surface was found to exhibit oscillatory motion and the flow regime was varied with time in the flow field. Wang et al. [31] carried out many simulation studies and discussions on the air flow characteristics and particle motion behaviors in impinging stream reactors with oscillatory dilute gas-solid jets. The influence of inlet flow oscillation frequency on the fluid motion and turbulence characteristics of the flow field have been deeply studied and examined. Ghadi and Esmailpour [32] performed many experimental investigations on the vortex structures and flow characteristics of the pulsed turbulent impinging stream reactor via the smoke-wire technique and high speed photography. Their results show that the flow regimes are varied due to inlet flow pulsation, and the pulsed turbulent impinging stream reactor can provide improvements in convective transport phenomena over the traditional impinging stream reactor. Liu et al [33] investigated and discussed flow and turbulence characteristics of impinging stream reactors with dynamic inlet velocity variation by CFD and Particle image velocimetry (PIV). They found that impinging surface makes back and forth motion in one period and inlet parameters have a great impact on the turbulence characteristics of impinging zone. To summarize the above literature review, these articles focus on the influence of dynamic inlet flow conditions, such as the inflow frequency and amplitude, on the flow and turbulence characteristics, generation, and motion characteristics of the vortex.

Despite extensive study on such systems, up to now, little information has been published concerning particle motion characteristics in the impinging stream reactor with dynamic inlet flow. In addition, little attention has been paid to the influence of the dynamic inflow conditions on the particle motions. In this work, we studied an impinging stream reactor with dynamic inlet flow, where both inlet velocity patterns exhibit step velocity variations with a half-period phase difference. The objective of this research was to investigate, by CFD-DEM, the effect of the variation period of the inlet velocity and velocity difference between the two inlet velocities on the particle motion behavior. The results of this work may help reveal how mean particle residence time is varied with different periods of inlet velocity variation and velocity difference between the two sides of inlet velocities. In addition, our results can explain why the particle residence time is very short in the traditional impinging stream reactor. The organization of the rest of the paper is as follows. Section 2 gives a brief introduction of the theoretical analysis and condition simulations of gas and particle motions in the flow field via CFD-DEM tools. Section 3 gives a detailed description on the fluid flow characteristics, analyzes and discusses the effects of the variation period of the inlet velocity and velocity difference between the two inlet velocities on the motion behaviors and mean residence time of single and multiple particles in the impinging stream reactor with dynamic inlet flow, compared with traditional impinging stream reactor with equal mean inlet velocity. Section 4 summarizes research work and research conclusions of this paper and next specific topics in future.

\section{Model Description}

A combined technique using computational fluid dynamics (CFD) and the discrete element method (DEM) with a soft-sphere model was used to study the particle and gas motion in a gas-solid two-phase impinging stream reactor with dynamic inlet flow. A realizable $k-\varepsilon$ model was chosen to measure the gas phase turbulence under the simulation conditions, as recommended by Choicharoen et al. [21]. 


\subsection{Gas Motion Equations}

The continuity and momentum balance equations, as well as a turbulence model without chemical reactions, are necessary to describe our system. The details are expressed as follows [6].

The continuity balance is given by:

$$
\frac{\partial \alpha \rho}{\partial t}+\frac{\partial}{\partial x_{i}}\left(\alpha \rho u_{i}\right)=S_{m}
$$

The momentum balance on the nozzle axis is:

$$
\frac{\partial}{\partial t}\left(\alpha \rho u_{j}\right)+\frac{\partial}{\partial x_{i}}\left(\alpha \rho u_{i} u_{j}\right)=-\alpha \frac{\partial p}{\partial x_{j}}+\frac{\partial}{\partial x_{i}}\left[\alpha\left(\mu+\mu_{t}\right)\left(\frac{\partial u_{i}}{\partial x_{j}}+\frac{\partial u_{j}}{\partial x_{i}}\right)\right]+\alpha \rho g_{i}+S_{f}
$$

A realizable $k-\varepsilon$ model is used to model the gas phase turbulence. The $k-\varepsilon$ equations can be expressed as follows [34]:

$$
\begin{gathered}
\frac{\partial(\alpha \rho k)}{\partial t}+\frac{\partial\left(\alpha \rho k u_{i}\right)}{\partial x_{i}}=\frac{\partial}{\partial x_{j}}\left[\alpha\left(\mu+\frac{\mu_{t}}{\sigma_{k}}\right) \frac{\partial k}{\partial x_{j}}\right]+\alpha G_{k} \\
\frac{\partial(\alpha \rho \varepsilon)}{\partial t}+\frac{\partial\left(\alpha \rho \varepsilon u_{i}\right)}{\partial x_{i}}=\frac{\partial}{\partial x_{j}}\left[\alpha\left(\mu+\frac{\mu_{t}}{\sigma_{\varepsilon}}\right) \frac{\partial \varepsilon}{\partial x_{j}}\right]-\alpha \rho\left(C_{1} S \varepsilon-C_{2} \frac{\varepsilon^{2}}{k+\sqrt{v \varepsilon}}\right),
\end{gathered}
$$

where $G_{k}=\mu_{t} S^{2}, C_{1}=\max [0.43, \eta /(\eta+5)], \eta=S k / \varepsilon, \mu_{t}=\rho C_{\mu} k^{2} / \varepsilon, C_{2}=1.9, \sigma_{\varepsilon}=1.2, \sigma_{k}=1.0$, and $S=\left(2 S_{i j} S_{i j}\right)^{1 / 2}$. In the above equations, $\alpha$ represents the void fraction, $\rho$ denotes the gas density, $u$ is the gas phase velocity, and $g_{i}$ is the $i$ th component of the gravitational acceleration. $S_{m}$ and $S_{f}$ represent source terms in the mass balance and momentum balance equations, respectively.

\subsection{Particle Motion Equations}

The motion equation of a single particle in the $x$ direction of the flow field is:

$$
\frac{d u_{p i}}{d t}=\frac{0.75 C_{f} \rho}{\rho_{p} d_{p}}\left|u_{p}-u\right|\left(u_{p i}-u_{i}\right)
$$

In this work, since the flow state of single particles in the impinging stream reactor are in the transition zone [35], the drag coefficient is expressed as follows:

$$
C_{f}=12.5 / \operatorname{Re}_{p}^{0.5}
$$

The motion balance of multiple particles on the nozzle axis is calculated according to Newton's second law. In addition, the particle motion and trajectory can be obtained by means of DEM. When there are many particles in the impinging stream reactor, both the drag force $F_{D}$ and the contact force $F_{C}$ are considered as external forces. The gravitational force $F_{g}$ acting on the particles is in the $z$ direction. In this study, $F_{C}$ is calculated via the soft-sphere model. Thus, the particle motion equation can be expressed as [36,37]:

$$
m_{p} \frac{d \vec{u}_{p}}{d t}=\vec{F}_{c}+\vec{F}_{D}+\vec{F}_{g}
$$

The drag force $F_{D}$ can be calculated according to the Ergun and Wen and Yu drag model [38], shown in Equations (8)-(11). $\beta$ represents interphase momentum exchange coefficient, based on the soft-sphere model:

$$
\vec{F}_{D}=\left[\frac{\beta}{1-\alpha}\left(\vec{u}-\vec{u}_{p}\right)\right] V_{p}
$$




$$
\begin{gathered}
\beta= \begin{cases}\frac{\mu(1-\alpha)}{\alpha d_{p}^{2}}\left[150(1-\alpha)+1.75 \operatorname{Re}_{p}\right] & (\alpha \leq 0.8) \\
\frac{3}{4} C_{f} \frac{\alpha(1-\alpha)}{d_{p}} \rho\left|\vec{u}_{p}-\vec{u}\right| \alpha^{-2.7} & (\alpha>0.8)\end{cases} \\
C_{f}= \begin{cases}24\left(1+0.15 \operatorname{Re}_{p}^{0.687}\right) / \operatorname{Re}_{p} & \left(\operatorname{Re}_{p} \leq 1000\right) \\
0.43 & \left(\operatorname{Re}_{p}>0\right)\end{cases} \\
\operatorname{Re}_{p}=\frac{\rho d_{p} \alpha\left|\vec{u}_{p}-\vec{u}\right|}{\mu}
\end{gathered}
$$

In the above equations, $F_{C}$ can be divided into two components, the normal component $\left(F_{c n}\right)$ and the tangential component $\left(F_{c t}\right)$. The expression of $F_{C}$ is shown in Equations (12)-(17). Not only interparticle collision but also the collision between particle and wall is considered in this work. $F_{c n, m r}$ and $F_{c t, m r}$ denote the normal and tangential contact force between particles " $m$ " and " $r$ ", respectively. $F_{c n, m r}$ and $F_{c t, m r}$ can be calculated by Equations (13), (15) and (17). Similarly, $F_{c n, m w}$ and $F_{c t, m w}$ represent the normal tangential contact force between particle " $m$ " and the wall, respectively. The expressions of $F_{c n, m w}$ and $F_{c t, m w}$ are given in Equations (14) and (16):

$$
\begin{gathered}
\vec{F}_{c}=\vec{F}_{c n}+\vec{F}_{c t} \\
\vec{F}_{c n, m r}=-\left(k_{n} \vec{\delta}_{n, m r}\right) \vec{n}_{m r}-\eta_{n}\left(\vec{u}_{p n, m r} \cdot \vec{n}_{m r}\right) \vec{n}_{m r} \\
\vec{F}_{c n, m w}=-\left(k_{n w} \vec{\delta}_{n, m w}\right) \vec{n}_{m}-\eta_{n w}\left(\vec{u}_{p n, m} \cdot \vec{n}_{m}\right) \vec{n}_{m} \\
\vec{F}_{c t, m r}=-k_{t} \vec{\delta}_{t, m r}-\eta_{t} \vec{u}_{p t, m r} \text { for }\left|\vec{F}_{c t, m r}\right| \leq f\left|\vec{F}_{c n, m r}\right| \\
\vec{F}_{c t, m w}=-k_{t w} \vec{\delta}_{t w}-\eta_{t w} \vec{u}_{p t, m} \text { for }\left|\vec{F}_{c t, m r}\right| \leq f\left|\vec{F}_{c n, m r}\right| \\
\vec{F}_{c t, m r}=f\left|\vec{F}_{c n, m r}\right| \vec{t}_{f o r}\left|\vec{F}_{c t, m r}\right|>f\left|\vec{F}_{c n, m r}\right| .
\end{gathered}
$$

Many experimental and numerical investigations indicate that most particles impinge with the trajectory particles in the impinging zone, and then scatter in all directions to the surroundings [28]. Only a few particles penetrate the opposite stream and undergo oscillatory motion. Short particle residence time greatly restricts the adequacy and integrity of the most relevant reactions, such as particle drying [6], mixing [9], desulfurization [7], etc.

\subsection{Model Assumptions and Simulation Conditions}

The following assumptions are adopted in this work. First, all particles have a uniform spherical shape and do not undergo any shape variations in all conditions. A three-dimensional computational domain is adopted to calculate the fluid flow characteristics and the geometry of the impinging stream reactor is comprised of two coaxial nozzles and one collision chamber, as shown in Figure 1. For the gas phase, the SIMPLE algorithm is adopted to solve the coupling between the gas pressure and velocity. The utilized gas phase time step is $1 \times 10^{-3} \mathrm{~s}$. For the particle phase, the particle motion equations are solved via an implicit discretization scheme. The utilized particle phase time step is $1 \times 10^{-6} \mathrm{~s}$, as suggested by Tsuji et al. [39]. The convergence criterion is set at $10^{-3}$ for all equations. The simulation conditions and properties of the gas phase are presented in Tables 1 and 2, respectively. The boundary conditions of impinging stream reactor are shown in Figure 1. The surroundings of the collision chamber and nozzle, and the exit of the collision chamber are modeled with the "wall" and "outflow" boundary conditions, respectively. 


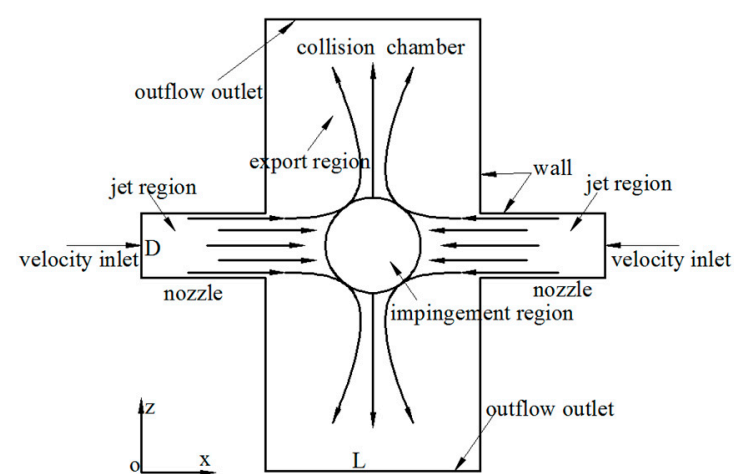

(a)

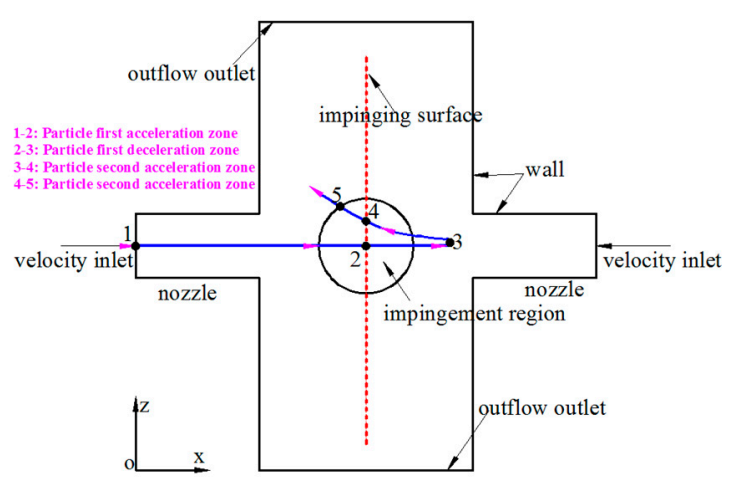

(b)

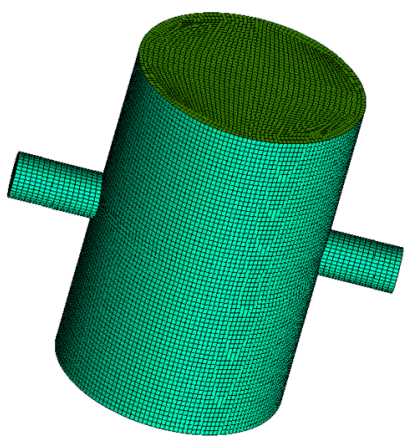

(c)

Figure 1. Geometric model of the impinging stream reactor: (a) $x-z$ plane; (b) particle motion zone; and (c) mesh model.

Table 1. Simulation conditions.

\begin{tabular}{|c|c|}
\hline Parameter & CFD Model \\
\hline Left inlet velocity $(\mathrm{m} / \mathrm{s})$ & $u_{1}=f_{1}(t)=\left\{\begin{array}{l}\mathrm{a}(0<t \leq 2 / T) \\
\mathrm{b}(2 / T<t \leq T)\end{array}\right.$ \\
\hline Right inlet velocity $(\mathrm{m} / \mathrm{s})$ & $u_{2}=f_{2}(t)=\left\{\begin{array}{l}\mathrm{b}(0<t \leq 2 / T) \\
\mathrm{a}(2 / T<t \leq T)\end{array}\right.$ \\
\hline$L / D$ & 5 \\
\hline Inlet velocity variation period (s) & $T=0.2,0.4,0.6,0.8,1.0,1.2,1.4,1.6$ \\
\hline Mean inlet velocity within one period (m/s) & $u_{m}=1$ \\
\hline Inlet velocity difference & $\Delta u=\mathrm{b}-\mathrm{a}, \mathrm{b}>\mathrm{a}$ \\
\hline Case 1 (traditional impinging stream, $u_{1}=u_{2}$ ) & $\mathrm{a}=\mathrm{b}=1 \mathrm{~m} / \mathrm{s}, \Delta u=0$ \\
\hline Case 2 & $\mathrm{a}=0.824 \mathrm{~m} / \mathrm{s}, \mathrm{b}=1.176 \mathrm{~m} / \mathrm{s}, \Delta u=0.352 \mathrm{~m} / \mathrm{s}$ \\
\hline Case 3 & $\mathrm{a}=0.889 \mathrm{~m} / \mathrm{s}, \mathrm{b}=1.111 \mathrm{~m} / \mathrm{s}, \Delta u=0.222 \mathrm{~m} / \mathrm{s}$ \\
\hline Case 4 & $\mathrm{a}=0.947 \mathrm{~m} / \mathrm{s}, \mathrm{b}=1.053 \mathrm{~m} / \mathrm{s}, \Delta u=0.106 \mathrm{~m} / \mathrm{s}$ \\
\hline Initial particle velocity $(\mathrm{m} / \mathrm{s})$ & $u_{p 0}=0$ \\
\hline Particle diameter $(\mathrm{m})$ & $d_{p}=5 \times 10^{-4}$ \\
\hline Particle density $\left(\mathrm{kg} / \mathrm{m}^{3}\right)$ & $\rho_{d}=1400$ \\
\hline Spring constant $(\mathrm{N} / \mathrm{m})$ & 1000 \\
\hline Friction coefficient & 0.4 \\
\hline Particle-particle restitution coefficient & 0.9 \\
\hline Particle-wall restitution coefficient & 0.9 \\
\hline
\end{tabular}


Table 2. Properties of the gas phase and geometric parameters of the impinging stream reactor.

\begin{tabular}{ccc}
\hline Property(Symbol) & Unit & Value \\
\hline density $(\rho)$ & $\mathrm{kg} / \mathrm{m}^{3}$ & 1.225 \\
viscosity $(\mu)$ & $\mathrm{kg} / \mathrm{m} \cdot \mathrm{s}$ & $1.7894 \times 10^{-5}$ \\
nozzle diameter $(D)$ & $\mathrm{mm}$ & 20 \\
\hline
\end{tabular}

From the abovementioned analysis, the single particle residence time is determined from the gas velocity distribution and the physical parameters of gas and particle phases. Multi-particles' mean residence time is not only determined by these factors, but also by the interparticle interactions in the impingement region. In this study, the velocity distribution variations of the gas phase are mainly caused by the dynamic inflow conditions of the impinging stream reactor. It can be seen in Table 1 that both left and right inlet velocities exhibit step variation in one period with a half-period phase shift in Cases 2-4. Case 1 represents traditional impinging stream $\left(u_{1}=u_{2}\right)$, while Cases $2-4$ represent impinging stream reactor with dynamic inlet flow. The mean inlet velocity within one period in Cases 2-4 are the same as that of Case 1, however, the inlet velocity differences in Cases 2-4 are different at the same period. The inlet velocity variations shown in Table 1 are interpreted and compiled by Fluent used define function (UDF), and then imported into Fluent for numerical calculation. Each simulation has been run for about twenty seconds to ensure a statistically-converged result.

To verify the independence of the grid models, 211,748, 253,526, 357,716, and 425,616 mesh elements are used to simulate the flow field. The stagnation point is a position where the fluid axial velocity is close to zero. The results in Table 3 show that the relative error in terms of both the turbulence kinetic energy and turbulence dissipation rate of the stagnation point are less than $3 \%$ in Case 1 when using grid numbers of 253,526, 357,716, and 425,616. Therefore, a 253,526 grid is chosen in all simulations to reduce the simulation time and ensure the accuracy of the simulation results.

Table 3. Grid independence tests in Case 1.

\begin{tabular}{ccccc}
\hline Grid Model & $\begin{array}{c}\text { Turbulence Kinetic } \\
\text { Energy of Stagnation } \\
\text { Point }\left(\mathbf{m}^{\mathbf{2}} \mathbf{s} \mathbf{s}^{\mathbf{}}\right)\end{array}$ & $\begin{array}{c}\text { Relative Error of } \\
\text { Turbulence } \\
\text { Kinetic Energy of } \\
\text { Stagnation Point }\end{array}$ & $\begin{array}{c}\text { Turbulence } \\
\text { Dissipation Rate of } \\
\text { Stagnation Point } \\
\left.\mathbf{( m}^{\mathbf{2}} \mathbf{/} \mathbf{s}^{\mathbf{3}}\right)\end{array}$ & $\begin{array}{c}\text { Relative Error of } \\
\text { Turbulence } \\
\text { Dissipation Rate of } \\
\text { Stagnation Point }\end{array}$ \\
\hline 211,748 & 0.128 & - & 1.501 & - \\
253,526 & 0.131 & $2.3 \%$ & 1.542 & $2.7 \%$ \\
357,716 & 0.132 & $0.75 \%$ & 1.540 & $0.12 \%$ \\
425,616 & 0.132 & 0 & 1.540 & 0 \\
\hline
\end{tabular}

\section{Results and Discussion}

\subsection{Gas Motion Behavior}

Figure 2 shows the velocity contour map of Case 1. It can be seen that the two opposite fluid impinging at the geometric center of the collision chamber, and the flow regime is steady and symmetric with respect to impinging surface at Case 1.

Figure 3 shows velocity contour maps of Case 2 with $T=0.6 \mathrm{~s}$. It can be seen from Figure 3 that the location of the impinging surface oscillates in the $x$-direction, due to the dynamic inflow conditions within one period. It can be inferred that impinging surface changes moving direction every half-period. The simulated gas motion behavior is consistent with the study of Erkoc [29] and Li [30]. A similar fluid transport phenomenon is also obtained in Cases 3 and 4 . In summary, the flow regime of Case 1 is steady and the impinging surface lies in the $x=0.1 \mathrm{~m}$ plane, while the flow regimes of Cases $2-4$ vary with time and the impinging surface undergoes oscillatory motion within one period. 


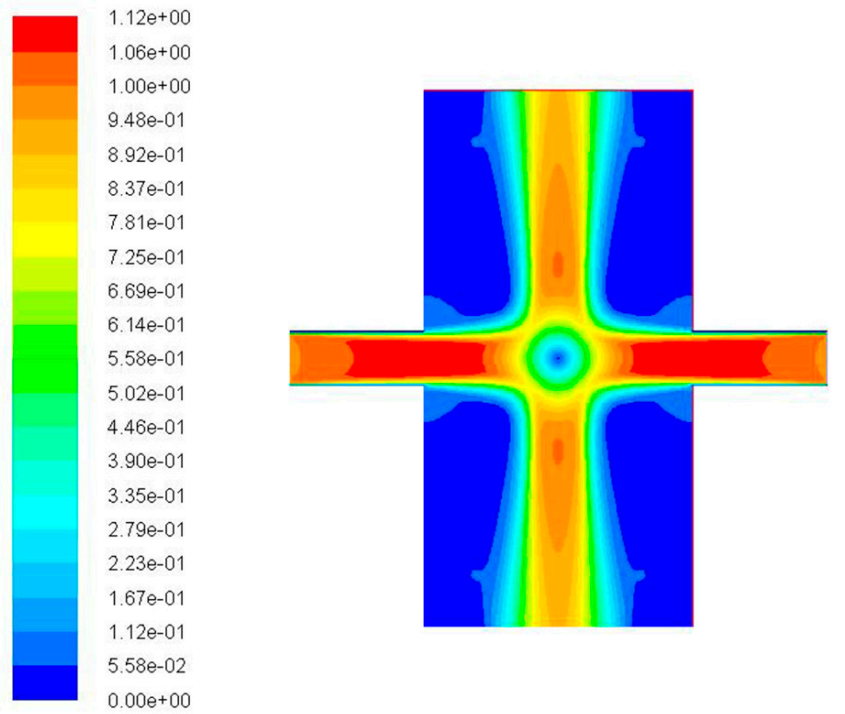

Figure 2. Velocity contour map at Case 1, unit in m/s.

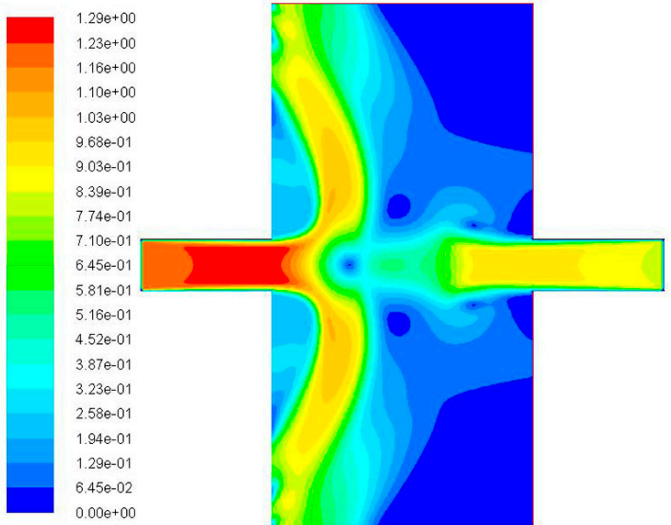

(a) $t=0.6 \mathrm{~s}$

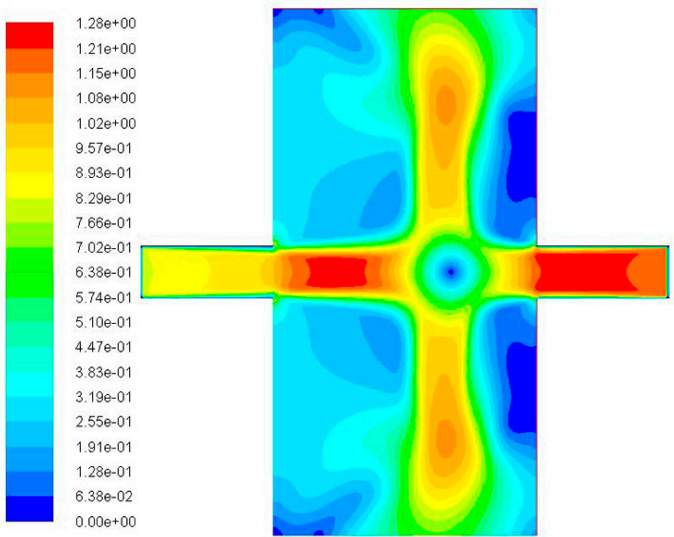

(c) $t=0.8 \mathrm{~s}$

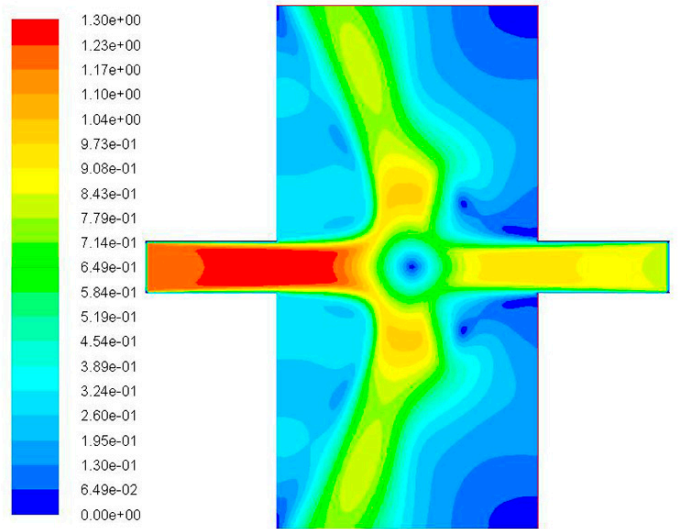

(b) $t=0.7 \mathrm{~s}$

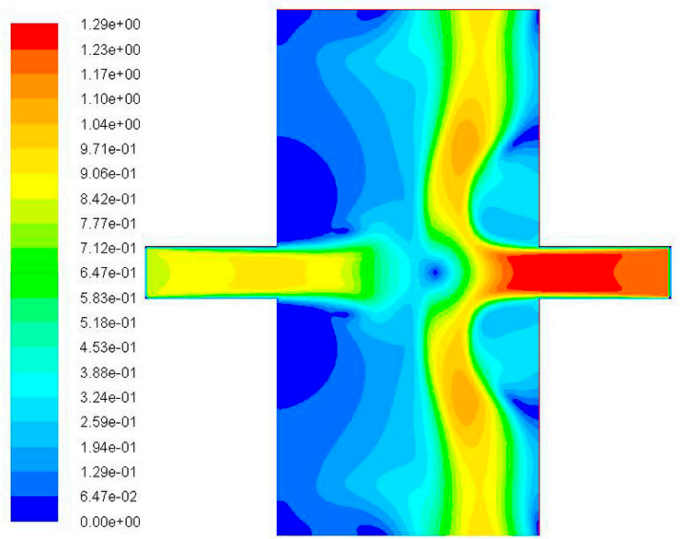

(d) $t=0.9 \mathrm{~s}$

Figure 3. Cont. 


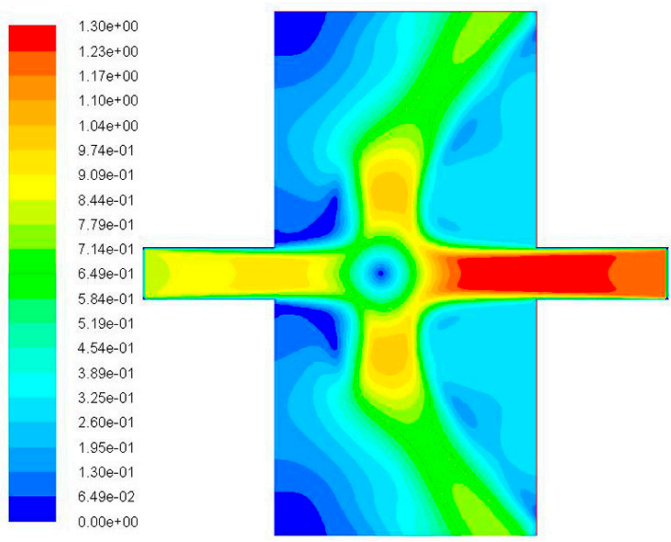

(e) $t=1.0 \mathrm{~s}$

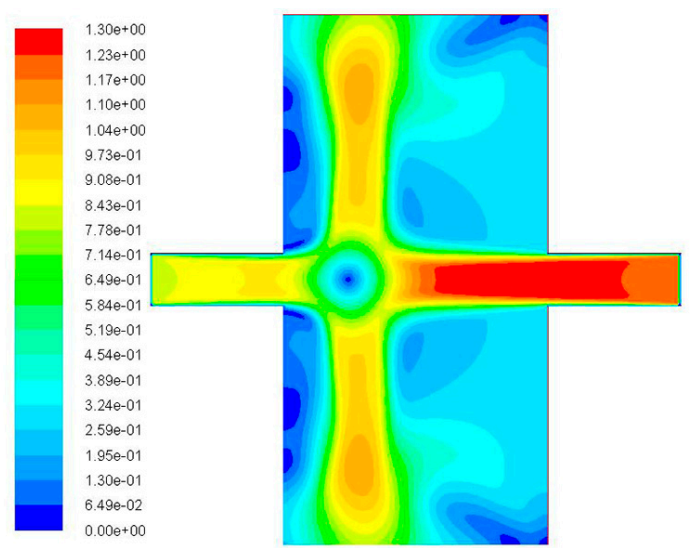

(f) $t=1.1 \mathrm{~s}$

Figure 3. Velocity contour maps at Case 2 and $T=0.6 \mathrm{~s}$, unit in $\mathrm{m} / \mathrm{s}$.

\subsection{Single Particle Motion Behavior}

Fluid flow characteristics play an important role in particle motions of the impinging stream. Since flow regimes vary with time and the impinging surface oscillates, it is of great significance to investigate and discuss the details of the particle motion characteristics in the impinging stream reactor with dynamic inlet flow.

Oscillatory motion of a single particle in the impinging stream reactor can significantly increase the relative velocity between the particle and gas, which can prolong the particle residence time in the active area. The active area consists of a jet region and an impingement region [1], as shown in Figure 1. Based on the above advantages, momentum, heat, and mass transfer in the active area can be greatly intensified. According to the particle motion characteristic shown in Figure 1b, at a single particle's $n$-th oscillation, the motion trajectory can be correspondingly divided into two parts: the $n$-th acceleration section and the $n$-th deceleration section. Therefore, based on Equations (5) and (6), the particle's motion equation for the $n$-th deceleration section can be expressed as:

$$
\begin{gathered}
\frac{d u_{p i, n}}{d t}=-k_{1}\left(u_{p i, n}+u_{i}\right)^{1.5}, k_{1}=0.75 \times 12.5 \times \frac{\mu^{0.5} \rho}{d_{p}^{1.5} \rho_{p}} \\
u_{p i, n}=\frac{d x_{i}}{d t}
\end{gathered}
$$

Equation (20) can be arranged by Equations (18) and (19). Thus, the particle motion equation of the $n$-th deceleration section can be also shown as follows:

$$
u_{p i, n} \frac{d u_{p i, n}}{d x_{i}}=-k_{1}\left(u_{p i, n}+u_{i}\right)^{1.5}
$$

After Equations (18) and (20) are integrated in the $n$-th deceleration section, $t_{d, n}$ and $x_{d, n}$ expressions are as follow:

$$
\begin{gathered}
t_{d, n}=\frac{2}{k_{1} u_{i, d, n}^{0.5}}\left[1-\left(1+\gamma_{d, n}\right)^{-0.5}\right] \\
x_{d, n}=\frac{2 u_{i, d, n}^{0.5}}{k_{1}}\left[\left(1+\gamma_{d, n}\right)^{0.5}-1\right]-\frac{2 u_{i, d, n}^{0.5}}{k_{1}}\left[1-\left(1+\gamma_{d, n}\right)^{-0.5}\right],
\end{gathered}
$$

where $\gamma_{d, n}$ represents the ratio of $u_{\text {pimax }, a c n}$ to $u_{i, d, n}$ in the $n$-th deceleration motion. Similarly, $t_{a c, n}$ and $x_{a c, n}$ expressions in the $n$-th acceleration section can be obtained, as shown below in Equations (23) and 
(24), respectively. While, $\gamma_{a c, n}$ represents the ratio of $u_{\text {pimax }, a c, n}$ to $u_{i, a c, n}$ in the $n$-th acceleration motion. i.e., $\gamma_{a c, n}=u_{p i, m a x}, a c, n / u_{i, a c, n}$ :

$$
\begin{gathered}
t_{a c, n}=\frac{2}{k_{1} u_{i, a c, n}^{0.5}}\left[\left(1-\gamma_{a c, n}\right)^{-0.5}-1\right] \\
x_{a c, n}=\frac{2 u_{i, a c, n}^{0.5}}{k_{1}}\left\{\left[\left(1-\gamma_{a c, n}\right)^{0.5}-1\right]+\left[\left(1-\gamma_{a c, n}\right)^{-0.5}-1\right]\right\} .
\end{gathered}
$$

For the traditional impinging stream reactor, $x_{a c, n+1}=x_{d, n}$ [2]. The relationship between $\gamma_{n}$ and $\gamma_{n+1}$ is as follows:

$$
\left[\left(1-\gamma_{n+1}\right)^{0.5}+\left(1-\gamma_{n+1}\right)^{-0.5}\right]-\left[\left(1+\gamma_{n}\right)^{0.5}+\left(1+\gamma_{n}\right)^{-0.5}\right]=0 .
$$

The parameter $\gamma_{n}$ is the ratio of the maximum axial velocity of the particle to the fluid axial velocity in the $n$-th period of oscillatory motion. In addition, $\gamma_{n}$ reflects the maximum kinetic energy obtained in the jet region and determines the particle motion distance and residence time in the active area, based on Equations (21)-(24).

Figure 4 shows the functional form of $\gamma_{n+1}$ on $\gamma_{n}$ in the traditional impinging stream reactor based on Equation (25). In Figure $4 \gamma_{n+1}$ increases when $\gamma_{n}$ increases, but $\gamma_{n+1}$ is weaker than $\gamma_{n}$ due to flow resistance. Thus, it can be determined from Figure 4 that both $\gamma_{n}$ and the particle's maximum axial kinetic energy $\left(0.5 u_{p i, \max }^{2}\right)$ greatly decrease (close to zero) after every oscillation. Finally, the particles leave the active area. It can be inferred that decreasing the particle maximum axial kinetic energy can shorten the particle residence time after every oscillatory motion.

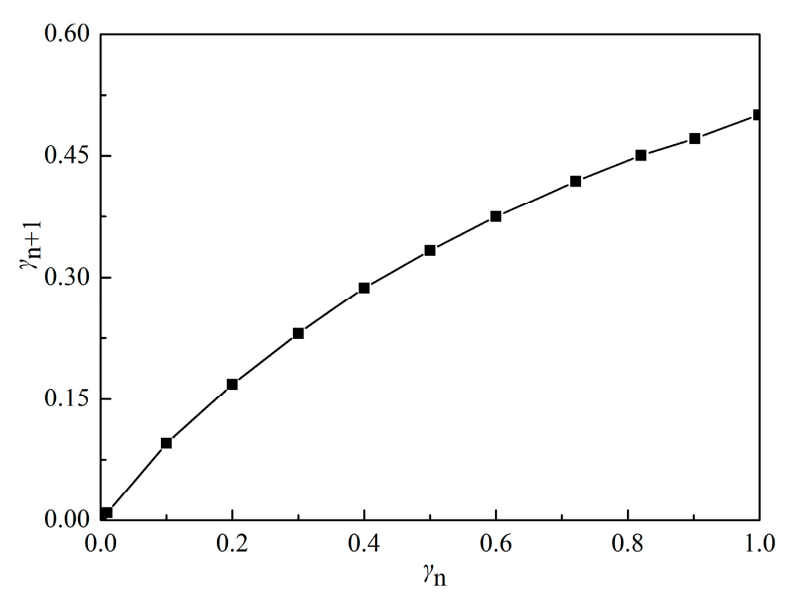

Figure 4. Functions of $\gamma_{n+1}$ on $\gamma_{n}$.

The residence time $t_{\text {tot }}$ and motion distance $x_{\text {tot }}$ are as follows:

$$
t_{t o t}=\sum t_{d, n}+\sum t_{a c, n}, x_{t o t}=\sum x_{d, n}+\sum x_{a c, n}
$$

where $t_{r}$ is the ratio of oscillatory motion time to $t_{a c, 1}$, and the expression of $t_{r}$ is shown as follows:

$$
t_{r}=\frac{t_{t o t}-t_{a c, 1}}{t_{a c, 1}}=\frac{t_{o s c}}{t_{a c, 1}}
$$

Based on this definition, $t_{r}$ reflects the size of the particle oscillation time given an equal first acceleration time. The parameter $t_{\text {tot }}$ increases with increasing $t_{r}$ at equal first particle acceleration time. The value of $t_{r}$ acts as a measure of the cost performance of the impinging stream reactor in terms of $t_{t o t}$, under the equal mean inlet velocity. 


\subsubsection{Single Particle Trajectory}

Figure 5 shows the single particle trajectory in the active area at Case 1, while Figure 6 shows the single particle trajectory in the active area at Case 2 with several different periods. In Figures 5 and $6 \mathrm{a}-\mathrm{c}$ $x_{t o t, T=0.6 \mathrm{~s}}>x_{t o t, T=0.4 \mathrm{~s}}>x_{t o t, T=0.2 \mathrm{~s}}>x_{\text {tot, Case } 1}$. This is probably because increasing the period makes the impinging surface deviate further from the center axis, which extends the particle acceleration and deceleration zones when $0<T \leq 0.6 \mathrm{~s}$.

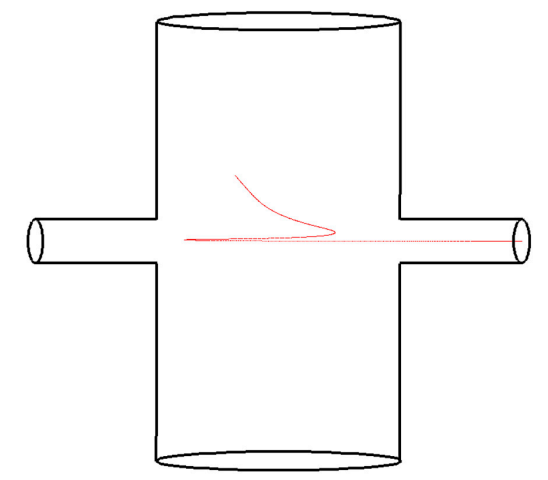

Figure 5. The single particle trajectory (red curve) in the active area at Case 1.

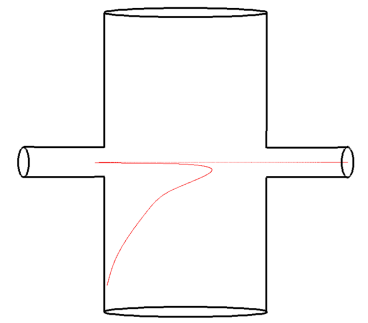

(a) $T=0.2 \mathrm{~s}$

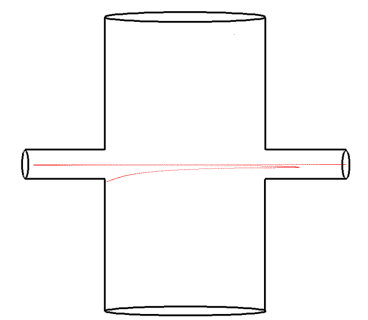

(e) $T=1.0 \mathrm{~s}$

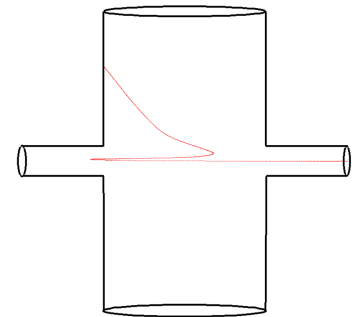

(b) $T=0.4 \mathrm{~s}$

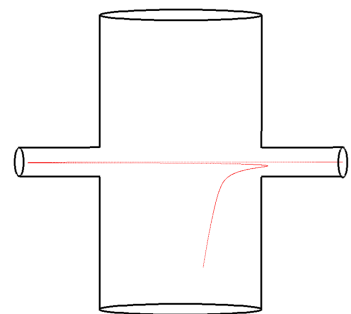

(f) $T=1.2 \mathrm{~s}$

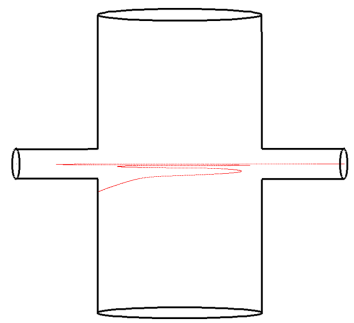

(c) $T=0.6 \mathrm{~s}$

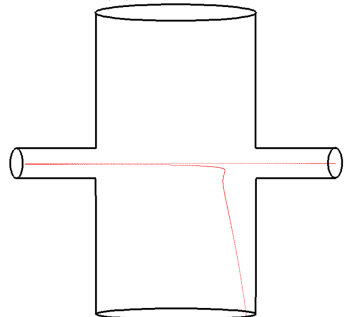

(g) $T=1.4 \mathrm{~s}$

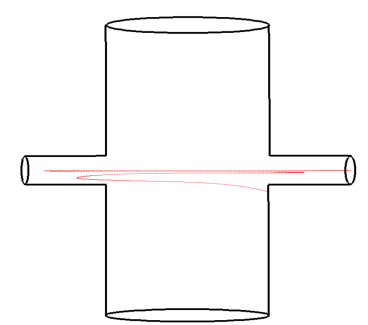

(d) $T=0.8 \mathrm{~s}$

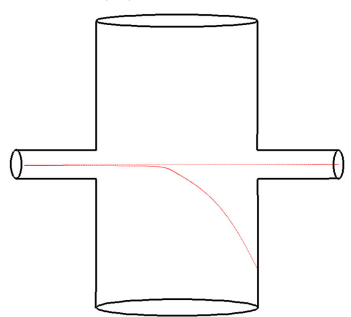

(h) $T=1.6 \mathrm{~s}$

Figure 6. The single particle trajectory (red curves) in the active area at Case 2 with several different periods.

In the first oscillatory motion of the asymmetric impinging stream reactor where $u_{1}=\mathrm{a}$ and $u_{2}=\mathrm{b} \neq \mathrm{a}$, at $t=t_{m a c, 1} \approx 0.3 \mathrm{~s}$ the particles' axial velocity reaches a maximum value, i.e., $u_{\text {pimax }, a c, 1}=u_{i}$, while at $t=t_{m d, 1} \approx 0.5 \mathrm{~s}$ the particle axial velocity reaches zero. Since the impinging surface moves very slowly after $t \geq t_{m a c, 1}$ when $T / 2 \geq t_{m a c, 1}$, the jet acceleration zone stays nearly the same and the flow regime basically remains constant. Thus, it is at $0.6 \leq T \leq 1.6 \mathrm{~s}$ that the first acceleration zone is the same. Additionally, instantaneously moving the impinging surface, changes the moving direction every half-period. For $T / 2=0.3 \mathrm{~s}$ or $0.4 \mathrm{~s}$, a single particle completes its first acceleration, and then flows into the right-moving impingement region where a large velocity gradient exists. This reduces the increasing degree of the radial velocity to a great extent, and radial velocity of the particle is close to zero at $T=0.6 \mathrm{~s}$ and $T=0.8 \mathrm{~s}$. In Figure $7 \mathrm{a}$ the radial particle velocities for $T=0.6 \mathrm{~s}$ and $T=0.8 \mathrm{~s}$ are much smaller than those of other periods in Case 2 after the third oscillatory motion. The results of Figure $7 \mathrm{~b}$ show that the number of oscillatory motions at $T=0.6 \mathrm{~s}$ and $T=0.8 \mathrm{~s}$ are larger than that of 
other periods in Case 2. Specifically, when $T / 2 \geq t_{m a c, 1}$, the particle makes oscillatory motions five times in Case 2 at $T=0.6 \mathrm{~s}$, four times at $T=0.8 \mathrm{~s}$, and three times at $T=1.0 \mathrm{~s}$ and in Case 1 . Thus, $x_{\text {tot }}$ increases with a decrease of $T$, as shown in Figure $6 \mathrm{c}-\mathrm{e}$.

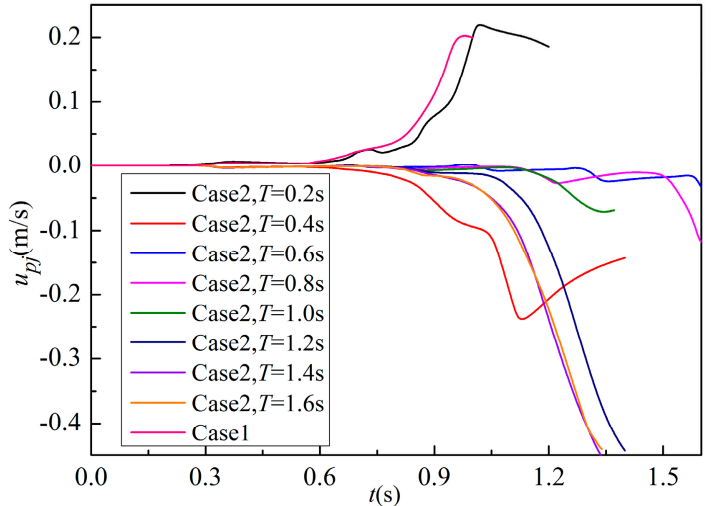

(a) $u_{p j}$

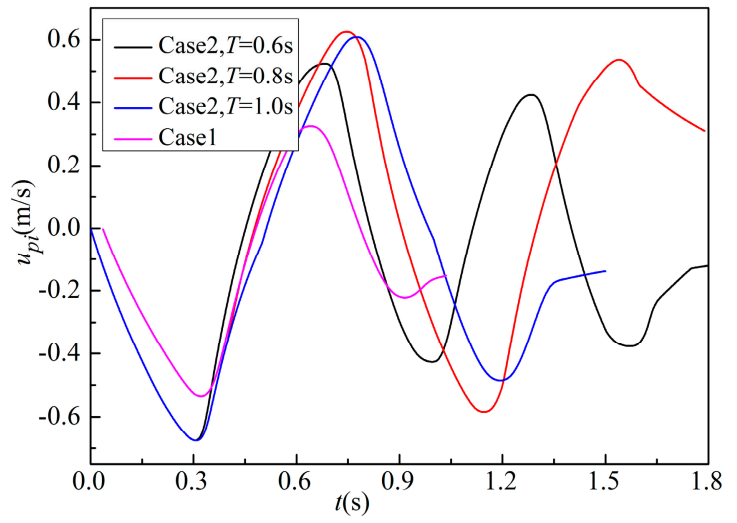

(b) $u_{p i}$

Figure 7. Particle radial (a) and axial (b) distribution.

Since a single particle completes its first oscillation at $t \approx t_{m d, 1}$ in the abovementioned asymmetric impinging stream reactor, the total of $x_{a c, 1}$ and $x_{d, 1}$ is almost equal when $1.0 \mathrm{~s} \leq T \leq 1.6 \mathrm{~s}$. Increasing $T$ shortens the particle second acceleration and deceleration zone for $1.0 \mathrm{~s} \leq T \leq 1.6 \mathrm{~s}$. Thus, $x_{\text {tot }}$ increases with a decrease of $T$, as shown in Figure 6e-h. Therefore, the total distance the particle moves decreases with an increase of $T$, when $T$ is larger than $t_{m a c, 1}$.

When the half-period of the inlet velocity variation is close to the particle's first acceleration time, i.e., $T / 2=t_{m a c, 1}$ and $T=0.6 \mathrm{~s}$, the particle axial velocity is equal to the fluid velocity. At the same time, changing the moving direction of the impinging surface can enhance the maximum axial velocity of the particle with every oscillatory motion, compared with the traditional impinging stream reactor with the same $u_{m}$. Furthermore, the radial particle velocity remains close to zero, and the particle can undergo oscillatory motion for more than three cycles. A similar particle motion characteristic can also be seen in Cases 3 and 4 . Therefore, the number of oscillatory motions and $x_{t o t}$ reach a maximum value at $T / 2=t_{m a c}, 1=0.3 \mathrm{~s}$ for Cases $2-4$.

\subsubsection{Single Particle Residence Time}

Generally, a single particle initially lying on the nozzle axis undergoes oscillatory motion three times, and the radial velocity greatly increases at last oscillating motion, which makes the particle quickly flow away from the active area. Figure 8 shows the particle residence time at different cases. It can be determined from Figure 8 that $t_{\text {tot }}, T=0.6 \mathrm{~s}>t_{\text {tot }}, T=0.4 \mathrm{~s}>t_{\text {tot, }} T=0.2 \mathrm{~s}>t_{\text {tot }}$, Case 1 . This is probably because the period increases with increasing maximum axial kinetic energy and prolongs the particle residence time in the opposite fluid, that is when $T / 2<t_{m a c, 1}=0.3 \mathrm{~s}$. From the abovementioned information, for $t_{m a c, 1} \leq T / 2 \leq t_{m d, 1}=0.5 \mathrm{~s}$, $t_{\text {tot }}$ increases with decreasing $T$ due to an increasing number of oscillatory motions. When $t_{m d, 1} \leq T / 2 \leq 0.8 \mathrm{~s}$, increasing $T$ can shorten the particle's second and third oscillatory motions. Thus, $t_{\text {tot }}$ decreases with increasing $T$ for $t_{m a c, 1} \leq T / 2 \leq 0.8 \mathrm{~s}$ and $t_{\text {tot }}$ reaches a maximum value at $T / 2=t_{m a c, 1}$. Therefore, the curve of Case 2 shown in Figure 8 accords with our theoretical expectations for the particle motion.

Similarly, the residence times of Cases 3 and 4, as shown in Figure 8, are basically the same as that of Case 2. That is, $t_{t o t}$ reaches a maximum value at $T / 2=t_{m a c, 1}=0.3 \mathrm{~s}$ in all cases. Increasing the inlet velocity difference $(\Delta u)$ can improve the maximum axial particle velocity in each oscillatory period and makes the particle remain longer in the active area, compared with the traditional impinging stream reactor under equal mean inlet velocity. Thus, $t_{\text {tot }}$ increases with an increase of $\Delta u$ at the same 
$T$. Overall, the particle residence time is closely related with period and inlet velocity difference of the dynamic inflow conditions.

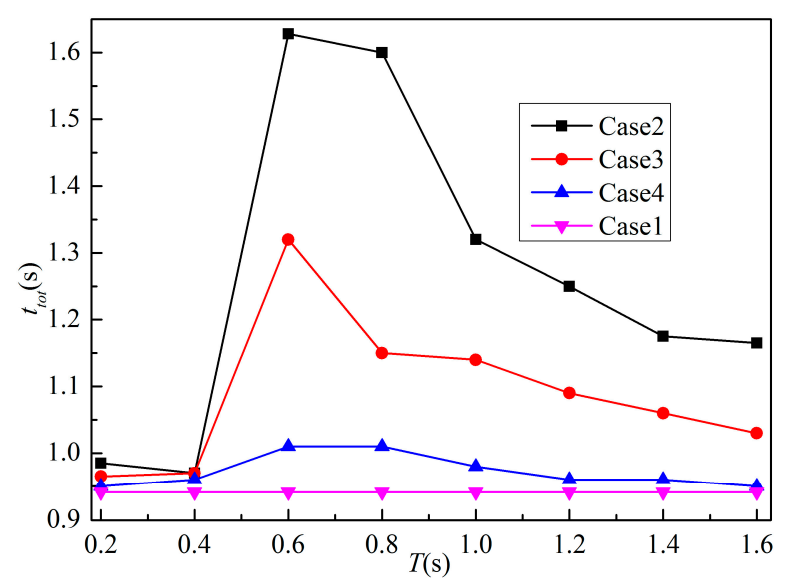

Figure 8. Particle residence time at different cases.

\subsubsection{Effects of $T$ and $\Delta u$ on the $t_{r}$}

Figure 9 shows the effect of $T$ on the $t_{r}$. Based on abovementioned analysis of the single particle residence time in different cases, $t_{\text {tot }}$ reaches a maximum value at $T / 2=t_{m a c, 1}=0.3 \mathrm{~s}$ in Cases 2-4, as shown in Figure 8. The first acceleration time $\left(t_{a c, 1}\right)$ has little change with different periods for the different cases. To normalize out this effect, we define $t_{r}$ as the ratio of the oscillatory time to $t_{a c, 1}$, which reflects the performance on the particle residence time at different $T$ and $\Delta u$ under the same mean inlet velocity. Based on the definition of $t_{r}, t_{r}$ reaches a maximum value at $T / 2=t_{a c, 1}$ in the different cases since the $t_{a c, 1}$ are approximately equal. Thus, the variation of $t_{r}$ (Figure 9) with $T$ is the similar to that of $t_{\text {tot }}$ (Figure 8). On the other hand, Figure 9 shows the effect of $\Delta u$ on $t_{r}$ for the different studied cases. The $t_{\text {tot }}$ increases with increasing $\Delta u$ at the same period, so $t_{r}$ increases with an increase of $\Delta u$. We can find from Figure 9 that the impinging stream reactor with dynamic inlet flow has a larger oscillatory time, i.e., larger $t_{r}$, over the traditional impinging stream reactor at equal $u_{m}$. Increasing $t_{r}$ means enhancing the particle's residence time in the active area of the impinging stream reactor. $t_{r}$ reaches a maximum value at $T / 2=t_{a c, 1}$. Therefore, the impinging stream reactor with dynamic inlet flow provides a higher cost performance over the traditional impinging stream reactor under equal $u_{m}$ in terms of the particle residence time.

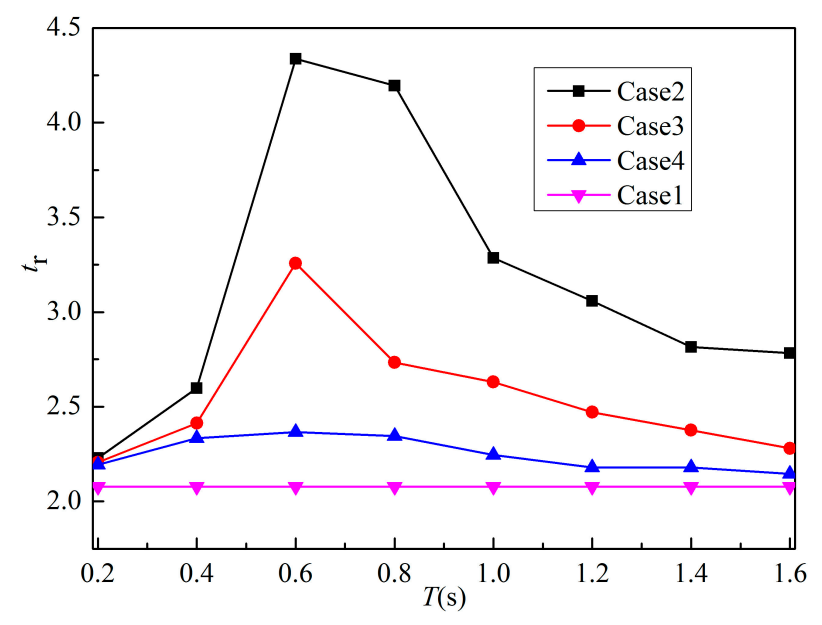

Figure 9. The effect of $T$ and $\Delta u$ on $t_{r}$. 


\subsection{Multi-Particle Motion Behavior}

\subsubsection{Multi-Particle Motion Characteristics}

Figure 10 shows the simulated particle motion behavior at different moments in Case 1 . It can be observed in Figure 10 that the highest particle concentration is in the impingement region, which is approximately axially symmetric with respect to the impinging surface. Few particles penetrate the reverse fluid to decelerate in Case 1. Both right and left particles accelerated by corresponding original jets frequently impinge with other particles in the vicinity of the impinging surface, and then spread away from the impingement center. This behavior is in accorded with the actual characteristics of multi-particles' motion in the traditional impinging stream reactor [40]. Meanwhile, due to the interparticle collisions, the mean particle's oscillation time trends to zero, i.e., $t_{o s c} \approx 0$. Based on the multi-phase transport phenomenon and the definitions of $t_{r}$, the interparticle collisions greatly shorten the particle's residence time. It can be inferred that most particles make no oscillatory motion in the active area due to collisions in the impingement region. Thus, interparticle collisions restrict the motion range of most particles to the scope of the impingement region, which greatly shortens the mean particle residence time compared with the single-particle motion characteristic shown in Figure 6. Therefore, the particles' frequent collisions in the impingement region improves the heat and mass transfer between particles [1], but limits the particle motion range and shortens the particle mean residence time in the traditional impinging stream reactor.

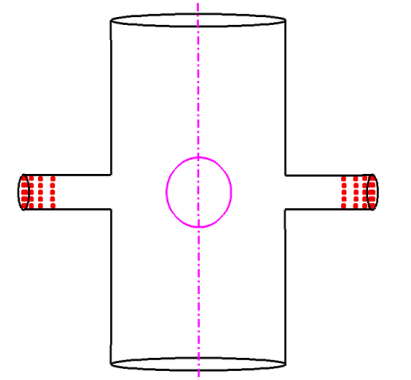

(a) $t=0.1 \mathrm{~s}$

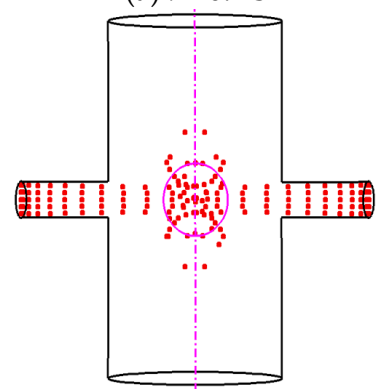

(d) $t=0.4 \mathrm{~s}$

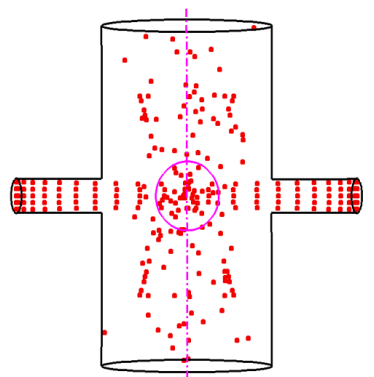

(g) $t=0.7 \mathrm{~s}$

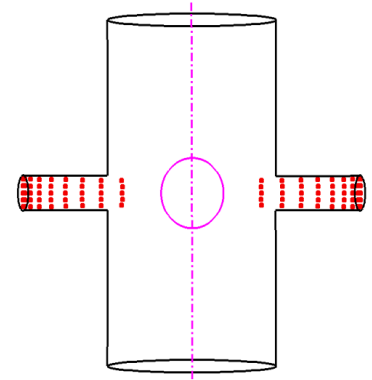

(b) $t=0.2 \mathrm{~s}$

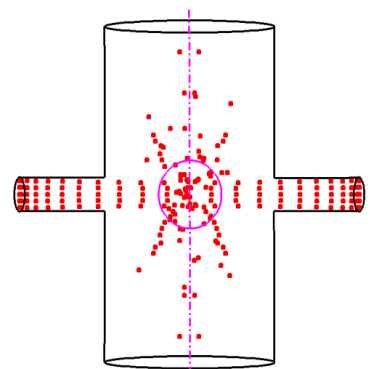

(e) $t=0.5 \mathrm{~s}$

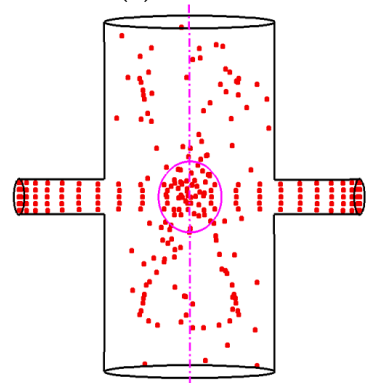

(h) $t=0.8 \mathrm{~s}$

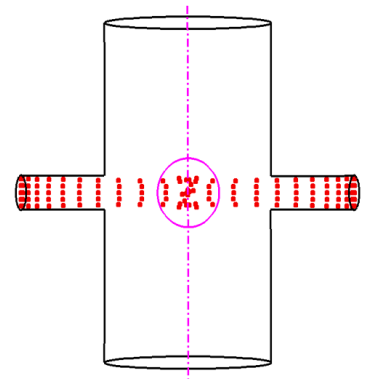

(c) $t=0.3 \mathrm{~s}$

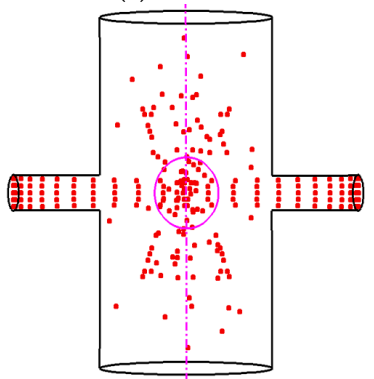

(f) $t=0.6 \mathrm{~s}$

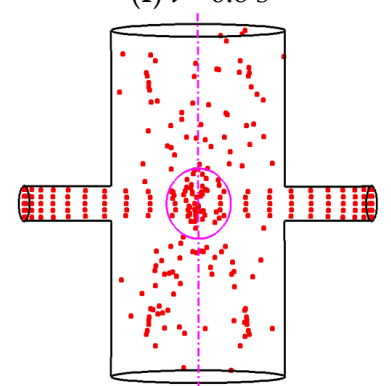

(i) $t=0.9 \mathrm{~s}$

Figure 10. Simulated particle motion at the indicated times for Case 1. The pink vertical dot-dash line and pink circle represent the impinging surface and impingement region, respectively. 
Figure 11 shows the simulated particle motion behavior at different moments for Case 2 and $T=0.6 \mathrm{~s}$. Similar to the multi-particles' motion characteristics for Case 1, the highest particle concentration is also in the impingement region. However, the axial particle concentration distribution is no longer symmetric with respect to the moving impinging surface due to the inlet velocity variation. Particle collisions occur anywhere in the flow field because the flow regime experiences dynamic changes, as shown in Figure 3. The instantaneously-moving impinging surface can cause several particles to be accelerated again by the fluid after collision, and thus undergo oscillatory motion at least once. From the single particle motion behavior in Case 2 (shown in Figure 7), we conclude that the range and number of oscillations are greatly reduced in multi-particle motion due to frequent particle collisions in the impingement region. Moreover, compared with multi-particle motion in Case 1, the ranges and number of oscillations in Case 2 increase because of the moving impinging surface of the flow field. Similar particle motion behavior is also observed in Cases 3 and 4 . Therefore, both multi-particles' motion ranges and the number of oscillations in the impinging stream reactor with dynamic inlet flow are larger than those of the traditional impinging stream reactor under equal $u_{m}$.

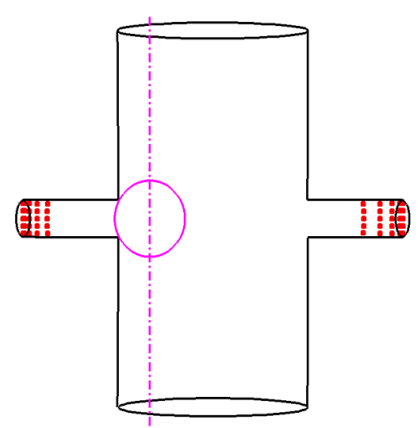

(a) $t=0.1 \mathrm{~s}$

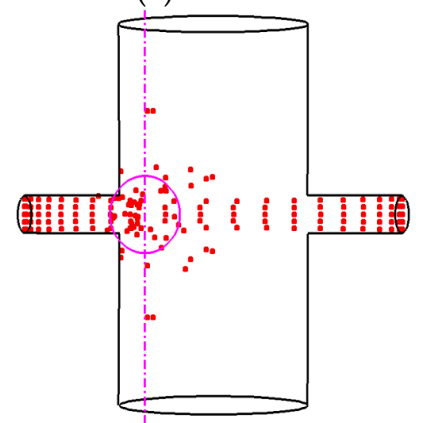

(d) $t=0.4 \mathrm{~s}$

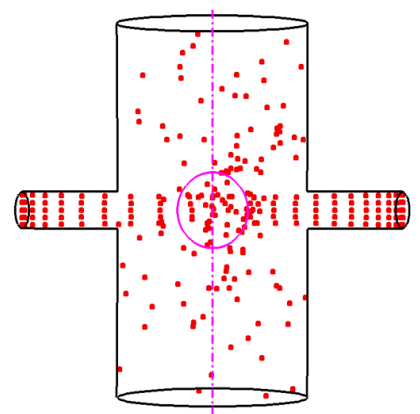

(g) $t=0.7 \mathrm{~s}$

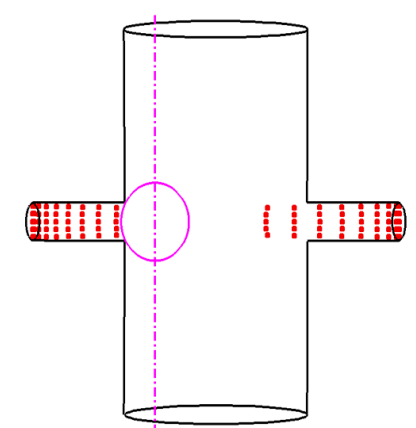

(b) $t=0.2 \mathrm{~s}$

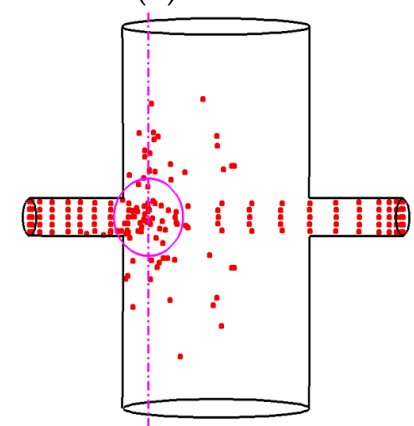

(e) $t=0.5 \mathrm{~s}$

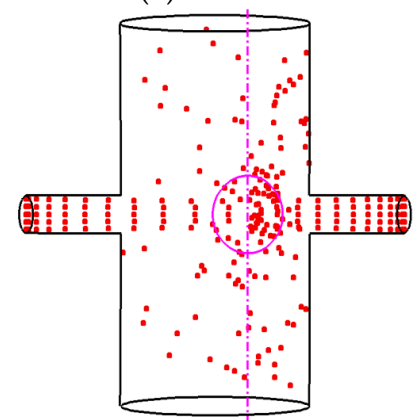

(h) $t=0.8 \mathrm{~s}$

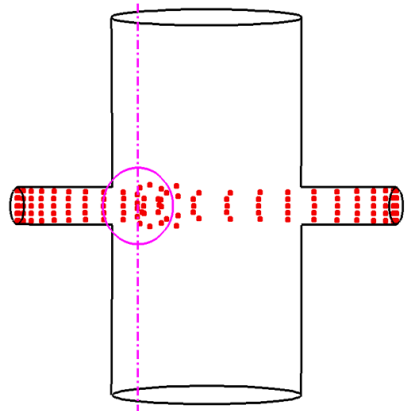

(c) $t=0.3 \mathrm{~s}$

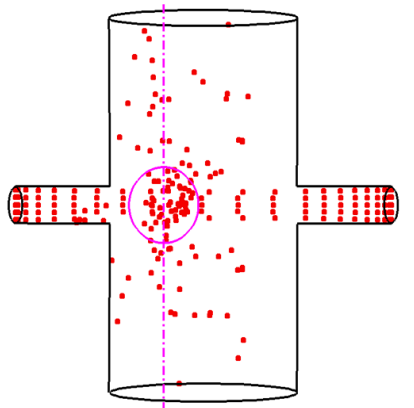

(f) $t=0.6 \mathrm{~s}$

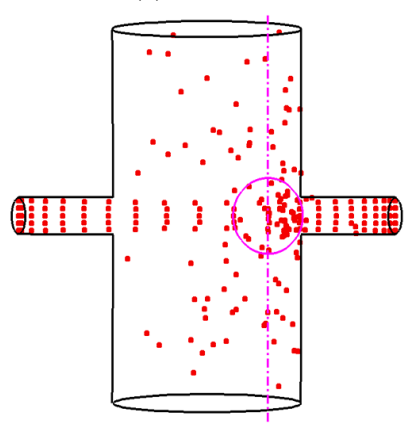

(i) $t=0.9 \mathrm{~s}$

Figure 11. Simulated particle motion at the indicated times for Case 2 and $T=0.6 \mathrm{~s}$. The pink vertical dot-dash line and pink circle represent the impinging surface and impingement region, respectively. 


\subsubsection{Multi-Particle Motion Model}

Figure 12 shows the simulated residence time distribution (RTD) of the particles calculated by means of the CFD-DEM model. RTDs are calculated from the residence time of 580 injected spherical particles. The RTD function is defined as the percentage of the particles leaving the active area during $c$ time intervals of length $\Delta t_{\text {min }}$. Thus, a general curve of the percentage of particles leaving the active area versus $c \Delta t_{\min }$ represents the RTD of the particles [2], where $c$ is the number of time intervals $(c=1,2,3, \ldots)$, and $\Delta t_{\min }$ is the minimum residence time of the particle in the active area. The peak of the RTD shifts towards the right with an increase of $T$ when $0<T<0.6 \mathrm{~s}$. This is probably because increasing the period $T$ of the dynamic inlet flow causes more particles to stay longer and extends the particle motion range in the active area. On the other hand, the peak of the RTD shifts towards the left with an increase of $T$ when $0.6 \mathrm{~s}<T<1.6 \mathrm{~s}$, due to the dynamic flow characteristics and interparticle collisions in the flow field. Next considering the dependence on the inlet velocity difference, the RTD shifts towards the right with an increase of $\Delta u$ for $T=0.6 \mathrm{~s}$, which indicates that increasing $\Delta u$ and the dynamic inflow conditions can continuously provide new kinetic energy to most of the particles during particle motion. The different RTDs indicate that $T$ and $\Delta u$ have a close relationship with the particle motion characteristics. Thus, it is necessary to measure and analyze the particle motion behaviors and mean particle residence time in the impinging stream reactor with dynamic inlet flow.

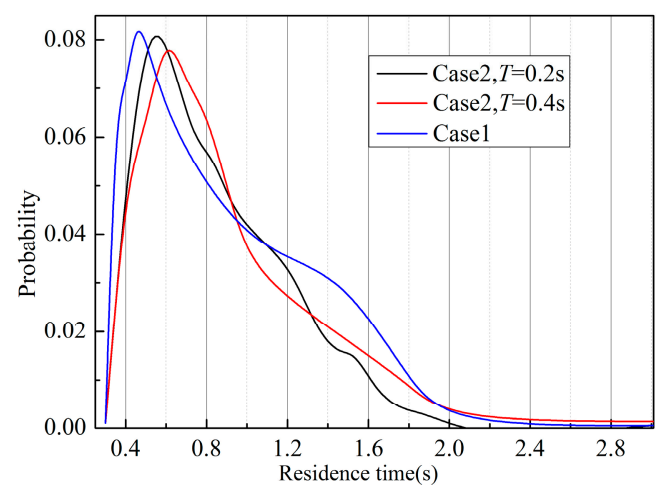

(a) Case 1, Case 2 at $T=0.2 \mathrm{~s}$ and $T=0.4 \mathrm{~s}$

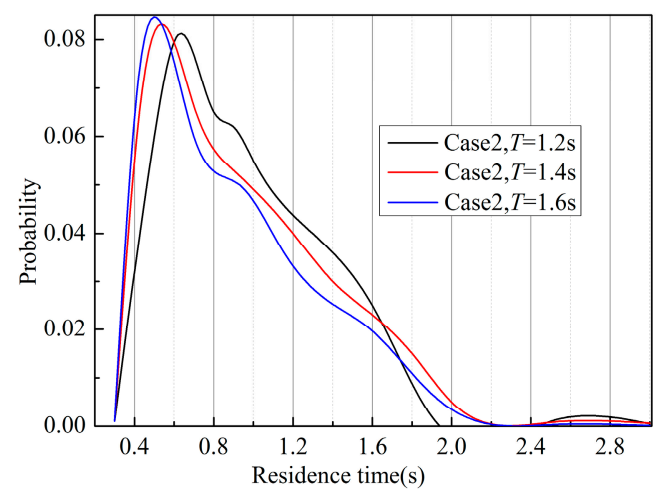

(c) Case 2 at $T=1.2 \mathrm{~s}, T=1.4 \mathrm{~s}$ and $T=1.6 \mathrm{~s}$

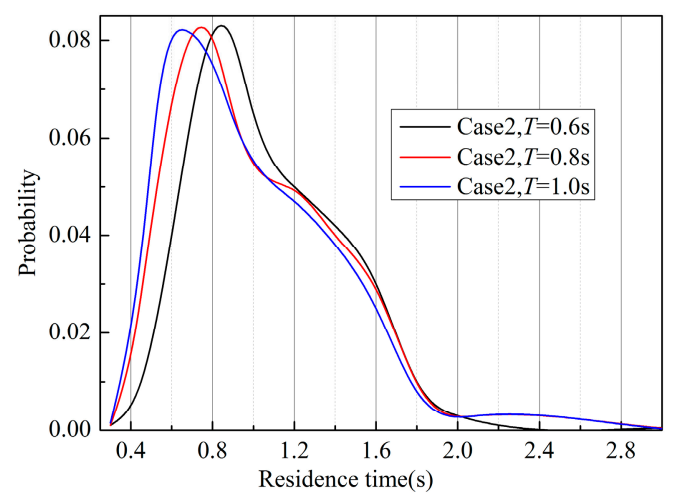

(b) Case 2 at $T=0.6 \mathrm{~s}, T=0.8 \mathrm{~s}$ and $T=1.0 \mathrm{~s}$

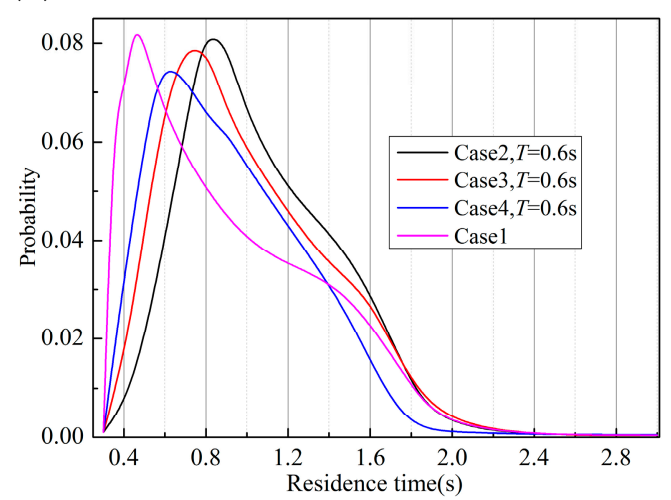

(d) Case 1 and Cases 2,3,4 at $T=0.6 \mathrm{~s}$

Figure 12. Simulated residence time distribution of particles.

Figure 13 shows common particle trajectories for Case 1. Both left and right particles impinge with each other in the impingement region, since the impingement region is stably located at the geometric center of the collision chamber in the traditional impinging stream reactor, as shown in Figure 3. Additionally, in Figure 13, the particles accelerated by the original fluid scatter in all directions after collisions. Most particles do not undergo oscillatory motion, but rather very fast deceleration in the original fluid, before quickly leaving the active area. 
Figure 14 presents common particle trajectories for Case 2. Due to the instantaneously moving impinging surface, the particle trajectories of Case 2 are much more complicated than those of Case 1 . It can be observed in Figures 10 and 11 that the particle motion range of Case 2 is larger than that of Case 1. Some particles accelerated by the large fluid velocity pass through the impinging surface, while the remainder of the particles accelerated by the weak fluid velocity reverse motion direction after particle collisions. At the same time, the impingement region is moving, which causes the corresponding particles to either be accelerated again by the original fluid or to enter the reverse fluid, resulting in oscillatory motion. However, a small number of particles directly move away from the active area due to the effect of the particles' collision on the enhancement of the radial particle velocity. Thus, in Figure 14a left particles' trajectories show that particles make no oscillatory motion. For the particles in Figure 14b, some particles are accelerated again by the original fluid after a collision and thus undergo oscillatory motion at least once. In Figure $14 \mathrm{c}, \mathrm{d}$ most of the right particles also undergo oscillatory motion at least once. This is probably because the particles pass through the impinging surface and then penetrate the reverse fluid, and even into the reverse nozzle before finally leaving the active area. As a result, the particle motion range can be greatly increased. Similar particle trajectories as those mentioned above can also be obtained in Cases 3 and 4 . Therefore, in terms of the particle trajectory, compared with the traditional impinging stream reactor under the same $u_{m}$, the complexity of particle trajectories increases. In addition, most particles undergo oscillatory motion at least once and the particle motion range is greatly enhanced, even filling the entire axial area of the collision chamber in the impinging stream reactor with dynamic inlet flow.

Based on these research results, the particle motion behavior of the impinging stream reactor with dynamic inlet flow are quite different from those of the traditional impinging stream reactor. It is of great importance to investigate and discuss the influence of the particles' dynamics and the different particle motion modes as shown in Figures 15-17. Such quantitative measures include the particle axial velocity and the effects of $T$ and inlet velocity difference $(\Delta u)$ on the mean particle residence time in the impinging stream reactor with dynamic inlet flow.

Figure 15 shows the axial particle velocity as a function of time for different motion modes. According to the particle trajectories of the impinging stream reactor with dynamic inlet flow shown in Figure 14, particle motion can be basically divided into six motion modes after the first interparticle collision in the impingement region. Mode 1, shown in Figure 15a, corresponds to a particle that directly leaves the active area due to the velocity fluctuation of the impingement region and the particle collision; Mode 2, shown in Figure 15a, corresponds to a particle that reverses motion direction and makes a short deceleration, before leaving the active area; Mode 3, shown in Figure 15a, corresponds to a particle that frequently impinges with other particles, and finally flows away from the active area; Mode 4, shown in Figure 15b, corresponds to a particle that reverses motion direction and makes a short deceleration, then is accelerated again by the original jet due to the moving impinging surface and undergoes several more collisions before finally leaving the active area; Mode 5, shown in Figure 15c, corresponds to a particle that penetrates the opposite fluid and decelerates, then is accelerated by the opposite fluid, reverses direction, and makes a short deceleration due to particle collisions in the reverse jet. Finally the particle flows away from the active area; and Mode 6, shown in Figure 15d, corresponds to a particle that penetrates the opposite fluid and moves into the impinging zone again, then undergoing several collisions in the impingement region, before finally leaving the active area. Thus, the different motion modes have a close relationships with the particle motion range and mean residence time in the impinging stream reactor. 


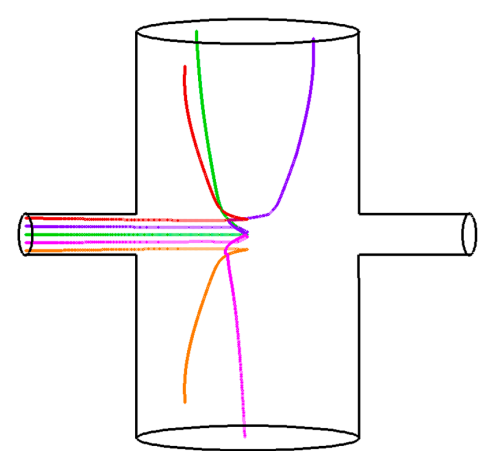

(a)

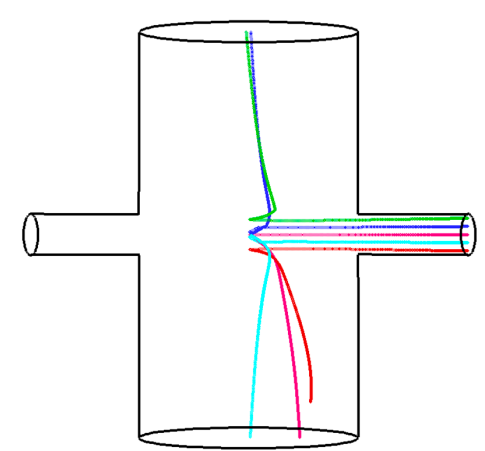

(b)

Figure 13. Common particle trajectories for Case 1: (a) left particles; and (b) right particles. Each color represents a different particle.

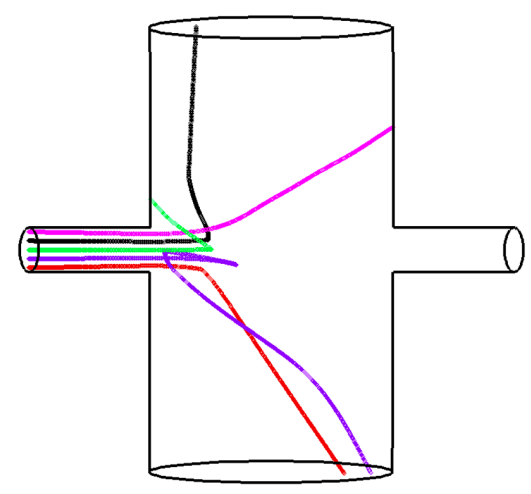

(a)

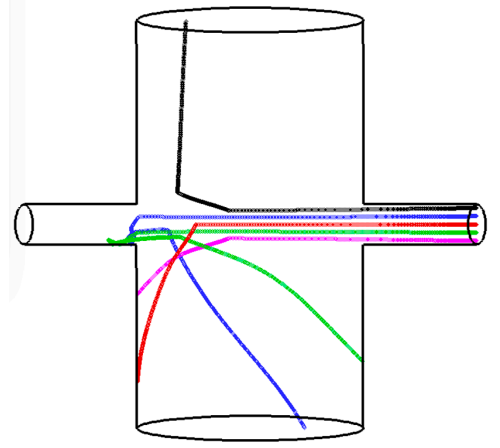

(c)

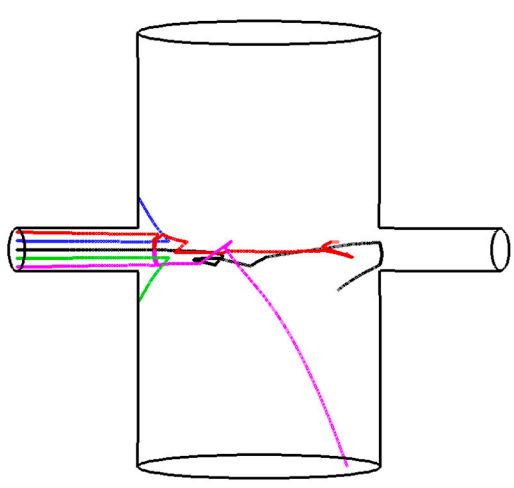

(b)

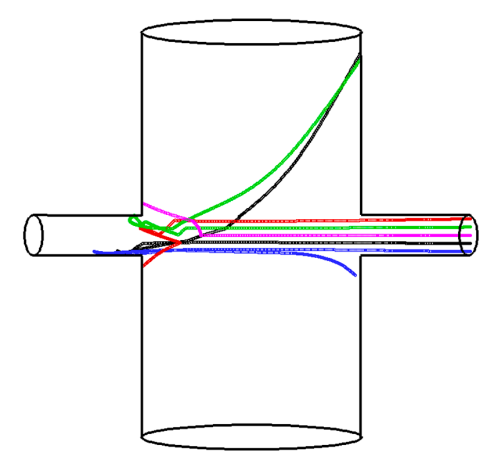

(d)

Figure 14. Common motion trajectories for Case 2: (a,b) characteristic left particles; and (c,d) characteristic right particles. Each color represents a different particle.

Figure 16 shows motion mode content in each motion mode for the different cases. Particle motion Modes 1-3 exist in the flow field and Mode 3 is the main particle motion behavior in the traditional impinging stream reactor. Thus, most particles do not oscillate and the particle mean residence time greatly decreases due to particle collisions in the impingement region. On the other hand, it can be observed in Figure 14 that particle motion from all six motion modes are observed in the impinging stream reactor with dynamic inlet flow. In Figure 16a particle motion Modes 1-3 still exist, in addition to the new Modes 4-6 for Cases 2-4. Additionally, motion Mode 5 is the main particle behavior for Cases $2-4$ with $T=0.6 \mathrm{~s}$. Motion Modes $4-6$ make the particle motion range and residence time increase 
because some particles can be accelerated again by the original or reverse fluid flows and thus undergo oscillatory motion at least once. Thus, increasing total content of Modes 4-6 can increase the particle motion range and residence time.

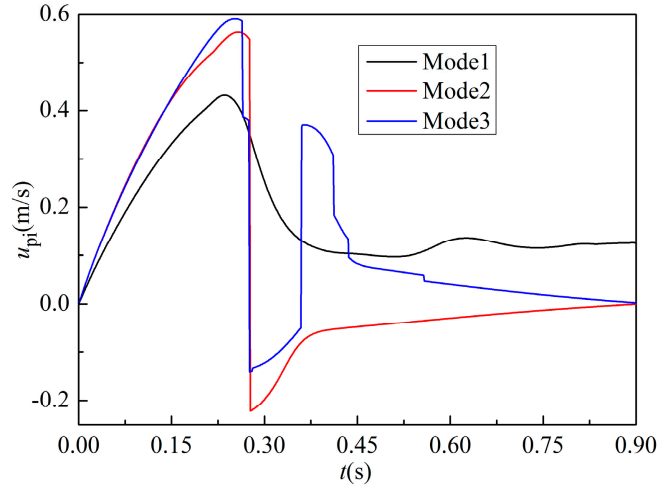

(a) Modes 1-3

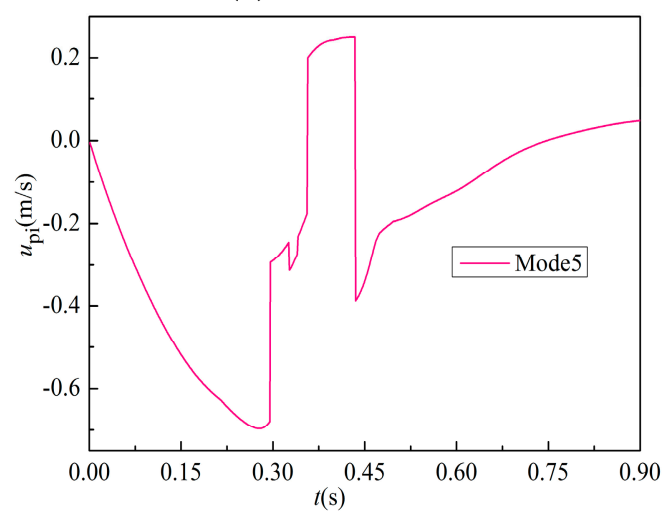

(c) Mode 5

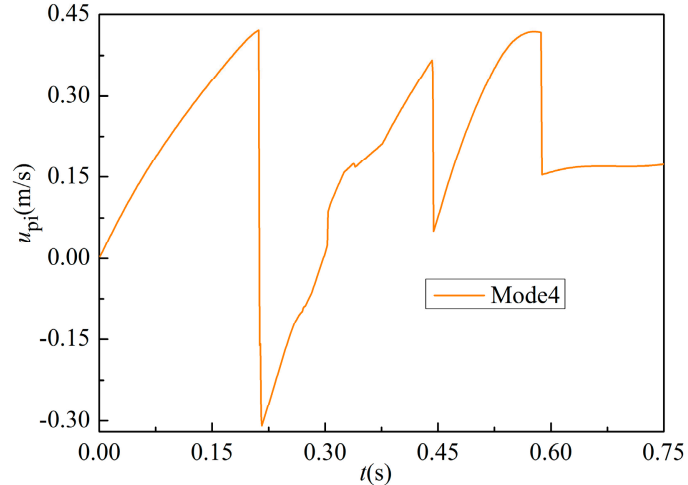

(b) Mode 4

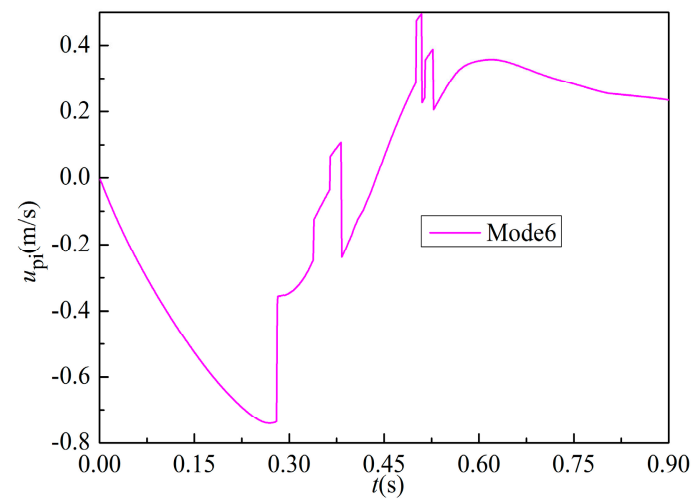

(d) Mode 6

Figure 15. Characteristic axial particle velocity as a function of time in the six different motion modes as labeled.

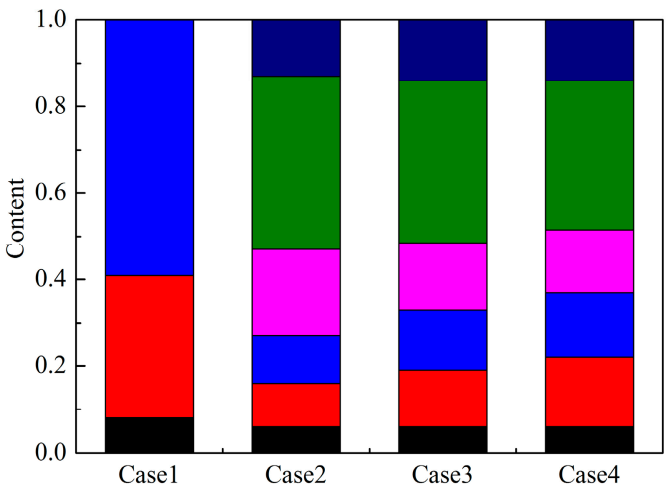

(a)

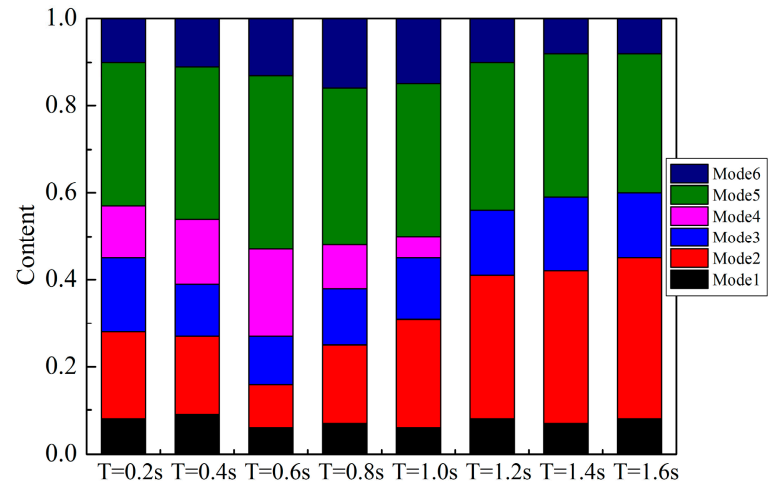

(b)

Figure 16. Motion mode content in each motion mode for the different cases; (a) Fixed $T=0.6 \mathrm{~s}$, and varying other cases; and (b) Case 2 while varying the inlet period $T$.

From the previous analysis, at $t=T / 2=t_{m a c, 1}, u_{p i}=u_{i}$ in the first oscillatory motion of the abovementioned asymmetric impinging stream reactor, where $u_{1}=\mathrm{a}$ and $u_{2}=\mathrm{b} \neq \mathrm{a}$. On the one hand, when $T / 2 \leq t_{m a c, 1}=0.3 \mathrm{~s}$, increasing $T$ can extend the particle acceleration zone and different particle behaviors are observed in motion Modes $4-6$. On the other hand, at $t_{m a c, 1}<T / 2 \leq t_{m d, 1}=0.5 \mathrm{~s}$, 
increasing $T$ shortens the particle's second acceleration zone. Thus, the total content of Modes 4-6 decreases with an increase of $T$. In addition, Mode 4 scarcely exists in Case 2 for $T / 2>t_{m d, 1}$, because the motion behavior of the impinging surface makes fewer particles accelerated again by fluid. Thus, Modes 2 and 5 are the main behaviors in Case 2 for $T / 2>t_{m d, 1}$. A similar mode content distribution at different $T$ can be also found in Cases 3 and 4 . In summary, the total content of Modes 4-6 increases with an increase of $T$ at $T / 2 \leq t_{m a c, 1}=0.3 \mathrm{~s}$ and decreases with an increase of $T$ at $T / 2>t_{m a c, 1}$.

\subsubsection{Mean Particle Residence Time}

Figure 17 shows the mean particle residence time for the different cases. The mean particle residence time of Cases 2-4 increase with an increase of $T$ for $T \leq 0.6 \mathrm{~s}$ and decrease with an increase of $T$ for $0.6 \mathrm{~s} \leq T \leq 1.8$ s, i.e., they reach a maximum value at $T / 2=t_{m a c, 1}=0.3 \mathrm{~s}$. This is probably because increasing the total content of Modes 4-6 can cause more particles to undergo oscillatory motion at least once and prolong the particle mean residence time. Moreover, the total content of Modes 4-6 increases with an increase of $T$ for $T / 2 \leq t_{m a c, 1}$ and decreases with an increase of $T$ for $t_{m a c, 1} \leq T / 2 \leq 0.8 \mathrm{~s}$, which is consistent with the variation of the mean particle residence time with $T$.

In addition, $t_{\text {tot }}$ increases with an increasing inlet velocity difference $(\Delta u)$ for the same $T$. This is probably because increasing $\Delta u$ leads to an increase of the maximum axial kinetic energy of the particle in particle motions in comparison with the traditional impinging stream. It can be determined from Figures 13-15 where the oscillations can be observed that number of oscillatory motion in Cases 2-4 is larger than those of Case 1, which means the mean particle residence time of Cases 2-4 is larger than that of Case 1. The aforementioned detailed information is consistent with the curves of RTD shown in Figure 12. Therefore, the mean particle residence time of the impinging stream reactor with dynamic inlet flow is larger than that of the traditional impinging stream reactor, under equal $u_{m}$. Overall, no matter whether single particle or multi-particles are employed in the impinging stream reactor, the mean particle residence time in the active area reaches a maximum value when half-period is close to the first acceleration time under dynamic inlet velocity variations with equal $u_{m}$.

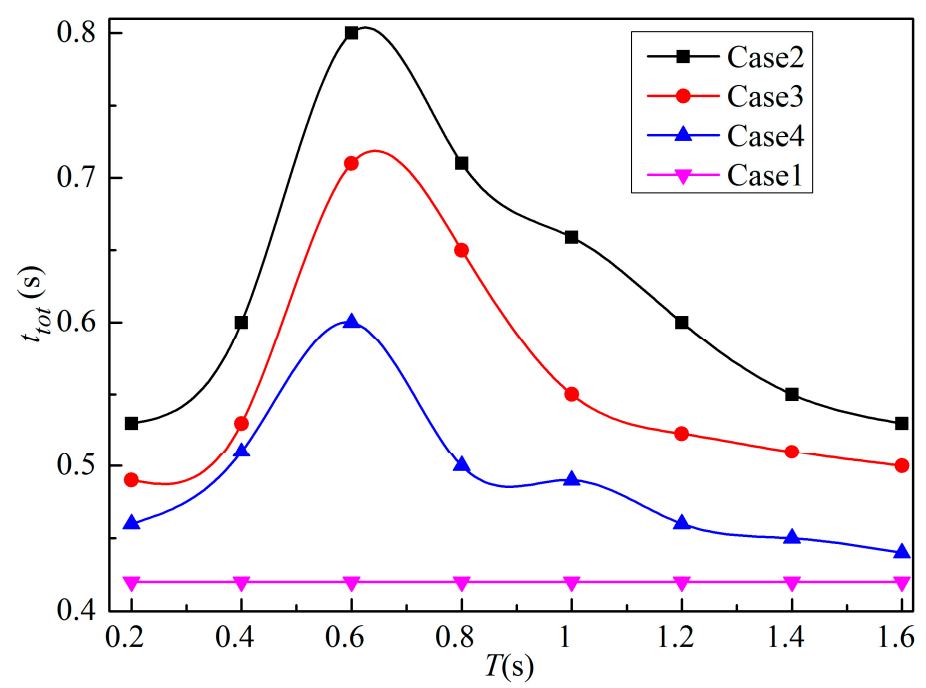

Figure 17. Mean particle residence time at different cases.

\section{Conclusions}

In this work, we performed many simulations on the flow characteristics and particle motion behavior of an impinging stream reactor with dynamic inflow conditions (both inlet velocity patterns exhibit step variation), compared with a traditional impinging stream reactor under equal mean inlet velocity $\left(u_{m}\right)$ over one period. Moreover, the effect of $T$ (variation period of the dynamic inlet 
flow) and $\Delta u$ (inlet velocity difference) on the single particle motion behavior, multi-particle motion characteristics, and the particle residence time were investigated and discussed. The following conclusions are drawn:

1. The impinging surface is no longer stably located at the geometric center of the collision chamber and instantaneously moves in the axial area. Additionally, the flow regime is varied with time in the impinging stream reactor with a dynamic inlet flow.

2. Single particle motion distance and residence time of the impinging stream reactor with a dynamic inlet flow are larger than those of a traditional impinging stream reactor under equal $u_{m}$. The ratio $t_{r}$ proposed in this work measures the particle oscillation time size under different conditions of the impinging stream reactor with equal $u_{m}$. Additionally, both $t_{r}$ and the residence time of a single particle reach a maximum value when half-period is approximately equal to the particle's first acceleration time, due to an increasing number of oscillatory motions. The impinging stream reactor with dynamic inlet flow provides a higher cost performance over the traditional impinging stream reactor under equal $u_{m}$, in terms of particle residence time.

3. Particle axial maximum kinetic energy greatly decreases because of flow resistance of the reverse fluid and interparticle collisions in the impinging stream reactor, which shortens the particle residence time and the motion range in the active area. Dynamic inflow conditions and increasing $\Delta u$ can continuously supply new energy to most particles and make more particles return to the active area again. Mean particle residence time and particle motion range increase with an increase of $\Delta u$ in the impinging stream reactor with dynamic inlet flow.

4. Based on the multi-particle motion characteristics, three new particle motion modes exist and differ from those of traditional impinging stream reactors. These new modes, where some particles are accelerated again by the original or reverse fluid flow, can cause some particles to perform oscillatory motion at least once in the active area.

5. The mean particle residence time of the impinging stream reactor with dynamic inlet flow is larger than that of the traditional impinging stream reactors under equal $u_{m}$ because of the three new particle motion modes, which account for the majority of particle behavior. Additionally, mean particle residence time reaches a maximum value when the half-period is approximately equal to the first particle acceleration time, because the maximum axial kinetic energy increases with each oscillatory motion and the number of oscillatory motions increases compared with the traditional impinging stream reactors. Studying the engineering applications of the impinging stream reactor with dynamic inlet flow will be conducted in future work.

Author Contributions: X.L. and S.Y. made numerical calculations and analyze data. X.L. wrote the original draft. L.L., W.G. and J.L. wrote review and edit the original draft.

Funding: This work was funded by the National Natural Science Foundation of China (Grant Nos. 51506072 and 51375183) and the National Basic Research Program of China (Project No. 2015CB251504).

Acknowledgments: This work was supported by the National Natural Science Foundation of China (Grant Nos. 51506072 and 51375183) and the National Basic Research Program of China (Project No. 2015CB251504).

Conflicts of Interest: The authors declare no conflict of interest.

Nomenclature

\begin{tabular}{lll}
\hline Symbol & Parameter Name & Unit \\
\hline$c$ & Number of time interval & - \\
$C_{f}$ & Drag coefficient & - \\
$D$ & Nozzle diameter & $\mathrm{m}$ \\
$f$ & Friction coefficient & - \\
$F_{C}$ & Contact force & $\mathrm{N}$ \\
$F_{c n}$ & Normal contact force & $\mathrm{N}$ \\
\hline
\end{tabular}




\begin{tabular}{|c|c|c|}
\hline Symbol & Parameter Name & Unit \\
\hline$F_{c t}$ & Tangential contact force & $\mathrm{N}$ \\
\hline$F_{D}$ & Frag force & $\mathrm{N}$ \\
\hline$F_{g}$ & Gravitational force & $\mathrm{N}$ \\
\hline$g_{i}$ & Gravitational acceleration component & $\mathrm{m} / \mathrm{s}^{2}$ \\
\hline$I$ & Turbulence intensity & - \\
\hline$k$ & Turbulence kinetic energy & $\mathrm{m}^{2} / \mathrm{s}^{2}$ \\
\hline$k_{n}$ & Spring constant in normal direction & $\mathrm{N} / \mathrm{m}$ \\
\hline$k_{t}$ & Spring constant in tangential direction & $\mathrm{N} / \mathrm{m}$ \\
\hline$L$ & Impinging spacing & $\mathrm{m}$ \\
\hline$m_{p}$ & Particle mass & $\mathrm{kg}$ \\
\hline$n$ & Number of oscillatory motion & - \\
\hline $\operatorname{Re}_{p}$ & Particle Reynolds number & - \\
\hline$S$ & Modulus of mean rate of strain tensor & - \\
\hline$S_{f}$ & source term in momentum balance equation & $\mathrm{N} / \mathrm{m}^{3}$ \\
\hline$S_{m}$ & source term in mass balance equation & $\mathrm{Kg} / \mathrm{m}^{3} \cdot \mathrm{s}$ \\
\hline$T$ & Inlet velocity variation period & $\mathrm{s}$ \\
\hline$\Delta t$ & Time step & $\mathrm{s}$ \\
\hline$\Delta t_{\min }$ & Minimum residence time of a particle in the active area & $\mathrm{s}$ \\
\hline$t_{a c, n}$ & Particle residence time in the $n$-th acceleration zone & $\mathrm{s}$ \\
\hline$t_{d, n}$ & Particle residence time in the $n$-th deceleration zone & $\mathrm{s}$ \\
\hline$t_{m a c, 1}$ & $t_{a c, 1}$ at $u_{1}=0.824 \mathrm{~m} / \mathrm{s}$ and $u_{2}=1.176 \mathrm{~m} / \mathrm{s}$ & $\mathrm{s}$ \\
\hline$t_{m d, 1}$ & $t_{d, 1}$ at $u_{1}=0.824 \mathrm{~m} / \mathrm{s}$ and $u_{2}=1.176 \mathrm{~m} / \mathrm{s}$ & $\mathrm{s}$ \\
\hline$t_{\text {osc }}$ & Particle oscillatory time, distance in the active area & $\mathrm{s}$ \\
\hline$t_{r}$ & The ratio of $t_{o s c}$ to $t_{t o t}$ & - \\
\hline$t_{\text {tot }}$ & Particle residence time in the active area & $\mathrm{s}$ \\
\hline$u, u_{i}, u_{j}$ & Gas velocity, velocity component & $\mathrm{m} / \mathrm{s}$ \\
\hline$\Delta u$ & Inlet velocity difference & $\mathrm{m} / \mathrm{s}$ \\
\hline$u_{1}, u_{2}$ & Left, right inlet velocity & $\mathrm{m} / \mathrm{s}$ \\
\hline$u_{m}$ & Mean inlet velocity within one period & $\mathrm{m} / \mathrm{s}$ \\
\hline$u_{p i}, u_{p j}$ & Particle velocity component & $\mathrm{m} / \mathrm{s}$ \\
\hline$u_{p i, n}, u_{p j, n}$ & Particle velocity component in the $n$-th oscillatory motion & $\mathrm{m} / \mathrm{s}$ \\
\hline$u_{\text {pimax }, n}$ & Maximum particle axial velocity in the $n$-th oscillatory motion & $\mathrm{m} / \mathrm{s}$ \\
\hline$u_{p n}$ & Relative velocity between particles in normal direction & $\mathrm{m} / \mathrm{s}$ \\
\hline$u_{p t}$ & Relative velocity between particles in tangential direction & $\mathrm{m} / \mathrm{s}$ \\
\hline$V_{p}$ & Volume of particle & $\mathrm{m}^{3}$ \\
\hline$x$ & Coordinate on the nozzle axis & $\mathrm{m}$ \\
\hline$x_{a c, n}$ & Particle motion distance in the $n$-th acceleration zone & $\mathrm{m}$ \\
\hline$x_{d, n}$ & Particle motion distance in the $n$-th deceleration zone & $\mathrm{m}$ \\
\hline$x_{i}, x_{j}$ & Coordinates & $\mathrm{m}$ \\
\hline$x_{\text {tot }}$ & Particle distance in the active area & $\mathrm{m}$ \\
\hline$\delta_{n}$ & Normal displacement & $\mathrm{m}$ \\
\hline$\delta_{t}$ & Tangential displacement & $\mathrm{m}$ \\
\hline$\eta_{n}$ & Damping coefficient in normal direction & $\mathrm{N} \cdot \mathrm{s} / \mathrm{m}$ \\
\hline$\eta_{t}$ & Damping coefficient in tangential direction & $\mathrm{N} \cdot \mathrm{s} / \mathrm{m}$ \\
\hline$\alpha$ & void fraction & - \\
\hline$\beta$ & Interphase momentum exchange coefficient & $\mathrm{kg} / \mathrm{m}^{3} \cdot \mathrm{s}$ \\
\hline$\gamma_{a c, n}$ & The ratio of $u_{\text {pimax,ac, } n}$ to $u_{i, a c, n}$ & - \\
\hline$\gamma_{d, n}$ & The ratio of $u_{\text {pimax }, a c, n}$ to $u_{i, d, n}$ & - \\
\hline$\gamma_{n}$ & Composed of $\gamma_{a c, n}$ and $\gamma_{d, n}$ in the $n$-th oscillatory motion & - \\
\hline$\varepsilon$ & Energy dissipation rate & $\mathrm{m}^{2} / \mathrm{s}^{3}$ \\
\hline
\end{tabular}




\begin{tabular}{lll}
\hline Symbol & Parameter Name & Unit \\
\hline$\mu$ & Dynamic viscosity & $\mathrm{kg} / \mathrm{m} \cdot \mathrm{s}$ \\
$\mu_{t}$ & Turbulent viscosity & $\mathrm{kg} / \mathrm{m} \cdot \mathrm{s}$ \\
$\rho$ & Gas density & $\mathrm{kg} / \mathrm{m}^{3}$ \\
$\sigma_{k}$ & Turbulent Prandtl number for $k$ & - \\
$\sigma_{\varepsilon}$ & Turbulent Prandtl number for $\varepsilon$ & - \\
$v$ & Kinematic viscosity & $\mathrm{m}^{2} / \mathrm{s}$ \\
\hline
\end{tabular}

\section{References}

1. Elperin, I.T. Heat and mass transfer in opposing currents. J. Eng. Phys. 1961, 6, 62-68.

2. Tamir, A. Impinging-Streams Reactors: Fundamentals and Applications; Elsevier: Amsterdam, The Netherlands, 1994.

3. Wu, H.; Pan, D.; Xiong, G.; Jiang, Y.; Yang, L.; Yang, B.; Peng, Z.; Hong, G. The abatement of fine particles from desulfurized flue gas by heterogeneous vapor condensation coupling two impinging streams. Chem. Eng. Process. 2016, 108, 174-180. [CrossRef]

4. Sedano-de la Rosa, C.; Vite-Torres, M.; Godínez-Salcedo, J.G.; Gallardo-Hernández, E.A.; Cuamatzi-Melendez, R.; Farfán-Cabrera, L.I. Erosion-corrosion of X-52 steel pipe under turbulent swirling impinging jets. Wear 2017, 376-377, 549-556. [CrossRef]

5. Du, M.; Gong, J.; Chen, W.; Wang, Q. Mathematical model based on DSMC method for particulate drying in a coaxial impinging streams dryer. Dry. Technol. 2015, 33, 646-658. [CrossRef]

6. Khomwachirakul, P.; Devahastin, S.; Swasdisevi, T.; Soponronnarit, S. Simulation of flow and drying characteristics of high-moisture particles in an impinging streams dryer via CFD-DEM. Dry. Technol. 2016, 34, 403-419. [CrossRef]

7. Wu, Y.; Li, Q.; Li, F. Desulfurization in the gas-continuous impinging streams gas-liquid reactor. Chem. Eng. Sci. 2007, 62, 1814-1824. [CrossRef]

8. Tamir, A.; Hershkovitz, D. Adsorption of $\mathrm{CO}_{2}$ in a new two-impinging-streams absorber. Chem. Eng. Sci. 1985, 40, 2149-2151. [CrossRef]

9. Bertrand, M.; Lamarque, N.; Lebaigue, O.; Plasari, E.; Ducros, F. Micromixing characterisation in rapid mixing devices by chemical methods and LES modelling. Chem. Eng. J. 2016, 283, 462-475. [CrossRef]

10. Krupa, K.; Nunes, M.I.; Ricardo, J.; Bourne, J.R. Characterization of micromixing in T-jet mixers. Chem. Eng. Sci. 2014, 111, 48-55. [CrossRef]

11. Metzger, L.; Kind, M. On the mixing in confined impinging jet mixers-Time scale analysis and scale-up using CFD coarse-graining methods. Chem. Eng. Res. Des. 2016, 109, 464-476. [CrossRef]

12. Zir, A.; Luzzatto, K.; Tamir, A. Application of free impinging streams to the combustion of gas and coal. Combust. Sci. Technol. 1988, 60,31-44.

13. Metzger, L.; Kind, M. The influence of mixing on fast precipitation processes-A coupled 3D CFD-PBE approach using the direct quadrature method of moments (DQMOM). Chem. Eng. Sci. 2017, 169, $284-298$. [CrossRef]

14. Saien, J.; Moradi, V. Low interfacial tension liquid-liquid extraction with impinging-jets contacting method: Influencing parameters and relationship. J. Ind. Eng. Chem. 2012, 18, 1293-1300. [CrossRef]

15. Dehkordi, A.M.; Kaghazchi, T.; Sohrabi, M. Application of an air-operated impinging streams contactor in liquid-liquid extraction. Chem. Eng. Technol. 2001, 24, 173-179. [CrossRef]

16. Berman, Y.; Tanklevsky, A.; Oren, Y.; Tamir, A. Modeling and experimental studies of $\mathrm{SO}_{2}$ absorption in coaxial cylinders with impinging streams: Part I. Chem. Eng. Sci. 2000, 55, 1009-1021. [CrossRef]

17. Berman, Y.; Tanklevsky, A.; Oren, Y.; Tamir, A. Modeling and Experimental studies of $\mathrm{SO}_{2}$ absorption in coaxial cylinders with impinging streams: Part II. Chem. Eng. Sci. 2000, 55, 1023-1028. [CrossRef]

18. Huai, X.L.; Peng, X.F.; Wang, G.X.; Liu, D.Y. Multi-phase flow and drying characteristics in a semi-circular impinging streams dryer. Int. J. Heat Mass Transf. 2003, 46, 3061-3067. [CrossRef]

19. Wang, S.J.; Mujumdar, A.S. Flow and mixing characteristics of multiple and multi-set opposing jets. Chem. Eng. Process. 2007, 46, 703-712. [CrossRef]

20. Liu, X.; Chen, Y.; Chen, Y. Analysis of gas-particle flow characteristics in impinging streams. Chem. Eng. Process 2014, 79, 14-22. [CrossRef] 
21. Choicharoen, K.; Devahastin, S.; Soponronnarit, S. Numerical simulation of multiphase transport phenomena during impinging streams drying of a particulate material. Dry. Technol. 2012, 30, 1227-1237. [CrossRef]

22. Li, W.-F.; Yao, T.-L.; Liu, H.-F.; Wang, F.-C. Experimental investigation of flow regimes of axisymmetric and planar opposed jets. AIChE J. 2011, 57, 1434-1446. [CrossRef]

23. Wu, D.; Li, J.; Liu, Z.; Xiong, Y.; Zheng, C.; Medwell, P.R. Eulerian and Lagrangian stagnation plane behavior of moderate Reynolds number round opposed-jets flow. Comput. Fluids 2016, 133, 116-128. [CrossRef]

24. Rieth, M.; Proch, F.; Stein, O.T.; Pettit, M.W.A.; Kempf, A.M. Comparison of the Sigma and Smagorinsky LES models for grid generated turbulence and a channel flow. Comput. Fluids 2014, 99, 172-181. [CrossRef]

25. Jafarikojour, M.; Sohrabi, M.; Royaee, S.J.; Rezaei, M. A new model for residence time distribution of impinging stream reactors using descending-sized stirred tanks in series. Chem. Eng. Res. Des. 2016, 109, 86-96. [CrossRef]

26. Rajaie, E.; Sohrabi, M. Application of the Monte Carlo technique in simulation of flow and modeling the residence time distribution in a continuous two impinging liquid-liquid streams contactor. Chem. Eng. J. 2008, 143, 249-256. [CrossRef]

27. Fatourehchi, N.; Sohrabi, M.; Dabir, B.; Royaee, S.J.; Haji Malayeri, A. Application of a novel type impinging stream reactor in solid-liquid enzyme reactions and modeling of residence time distribution using GDB model. Enzym. Microb. Technol. 2014, 55, 14-20. [CrossRef] [PubMed]

28. Du, M.; Zhao, C.; Zhou, B.; Guo, H.; Hao, Y. A modified DSMC method for simulating gas-particle two-phase impinging streams. Chem. Eng. Sci. 2011, 66, 4922-4931. [CrossRef]

29. Erkoc, E.; Fonte, C.P.; Dias, M.M.; Lopes, J.C.B.; Santos, R.J. Numerical study of active mixing over a dynamic flow field in a T-jets mixer-Induction of resonance. Chem. Eng. Res. Des. 2016, 106, 74-91. [CrossRef]

30. Li, W.-F.; Huang, G.-F.; Tu, G.-Y.; Liu, H.-F.; Wang, F.-C. Experimental study of oscillation of axisymmetric turbulent opposed jets with modulated airflow. AIChE J. 2013, 59, 4828-4838. [CrossRef]

31. Wang, S.; Li, X.; Fang, J.; Zhao, J.; Liu, L.; Liu, Y.; Liu, Y. Simulations of flow behavior of oscillatory opposed dilute gas-solid jets. Powder Technol. 2015, 284, 595-603. [CrossRef]

32. Ghadi, S.; Esmailpour, K.; Hosseinalipour, S.M.; Mujumdar, A. Experimental study of formation and development of coherent vertical structures in pulsed turbulent impinging jet. Exp. Therm. Fluid Sci. 2016, 74, 382-389. [CrossRef]

33. Liu, X.Q.; Yue, S.; Lu, L.Y.; Gao, W.; Li, J.L. Experimental and numerical studies on flow and turbulence characteristics of impinging stream reactors with dynamic inlet velocity variation. Energies 2018, 11, 1717. [CrossRef]

34. Wu, C.; Cheng, Y.; Ding, Y.; Jin, Y. CFD-DEM simulation of gas-solid reacting flows in fluid catalytic cracking (FCC) process. Chem. Eng. Sci. 2010, 65, 542-549. [CrossRef]

35. Enyakin, Y.P. Depth of penetration of solid- or liquid-phase particles in opposing gas-suspension jets. J. Eng. Phys. 1968, 14, 512-514. [CrossRef]

36. Sae-Heng, S.; Swasdisevi, T.; Amornkitbamrung, M. Investigation of temperature distribution and heat transfer in fluidized bed using a combined CFD-DEM model. Dry. Technol. 2011, 29, 697-708. [CrossRef]

37. ANSYS Inc. ANSYS Fluent Theory Guide; ANSYS Inc.: Canonsburg, PA, USA, 2013.

38. Zhong, W.; Xiong, Y.; Yuan, Z.; Zhang, M. DEM simulation of gas-solid flow behaviors in spout-fluid bed. Chem. Eng. Sci. 2006, 61, 1571-1584. [CrossRef]

39. Tsuji, Y.; Kawaguchi, T.; Tanaka, T. Discrete particle simulation of two-dimensional fluidized bed. Powder Technol. 1993, 77, 79-87. [CrossRef]

40. Welty, J.R.; Wicks, C.E.; Wilson, R.E. Fundamentals of Heat, Mass and Momentum Transfer; John Wiley and Sons: Hoboken, NJ, USA, 1969.

(C) 2018 by the authors. Licensee MDPI, Basel, Switzerland. This article is an open access article distributed under the terms and conditions of the Creative Commons Attribution (CC BY) license (http://creativecommons.org/licenses/by/4.0/). 